\title{
Lectures on the Topological Vertex
}

\author{
M. Mariño* \\ Department of Physics, Theory Division, CERN, University of Geneva \\ 1211 Geneva, Switzerland \\ marcos@mail.cern.ch \\ Marcos.Marino.Beiras@cern.ch
}

\section{Introduction and Overview}

The theory of Gromov-Witten invariants was largely motivated by the study of string theory on Calabi-Yau manifolds, and has now developed into one of the most dynamic fields of algebraic geometry. During the last years there has been enormous progress in the development of the theory and of its computational techniques. Roughly speaking, and restricting ourselves to Calabi-Yau threefolds, we have the following mathematical approaches to the computation of Gromov-Witten invariants:

1. Localization. This was first proposed by Kontsevich, and requires torus actions in the Calabi-Yau in order to work. Localization provides a priori a complete solution of the theory on toric (hence non-compact) CalabiYau manifolds, and reduces the computation of Gromov-Witten invariants to the calculation of Hodge integrals in Deligne-Mumford moduli space. Localization techniques make also possible to solve the theory at genus zero on a wide class of compact manifolds, see for example Cox and Katz (1999) for a review.

2. Deformation and topological approach. This has been developed more recently and relies on relative Gromov-Witten invariants. It provides a cutand-paste approach to the calculation of the invariants and seems to be the most powerful approach to higher genus Gromov-Witten invariants in the compact case.

3. D-brane moduli spaces. Gromov-Witten invariants can be reformulated in terms of the so-called Gopakumar-Vafa invariants (see Hori et al. (2003) for a summary of these). Heuristic techniques to compute them in terms of Euler characteristics of moduli space of embedded surfaces, and one can recover to a large extent the original information of Gromov-Witten theory. The equivalence between these two invariants remains however

\footnotetext{
* Also at Departamento de Matemática, IST, Lisboa, Portugal

K. Behrend, M. Manetti (eds.), Enumerative Invariants in Algebraic Geometry and String Theory. Lecture Notes in Mathematics 1947,

(C) Springer-Verlag Berlin Heidelberg 2008
} 
conjectural, and a general, rigorous definition of the Gopakumar-Vafa invariants in terms of appropriate moduli spaces is still not known. There is another set of invariants, the so-called Donaldson-Thomas invariants, that are also related to D-brane moduli spaces, which can be rigorously defined and have been conjectured to be equivalent to Gromov-Witten invariants by Maulik, Nekrasov, Okounkov and Pandharipande (2003).

Gromov-Witten invariants are closely related to string theory. It turns out that type IIA theory on a Calabi-Yau manifold $X$ leads to a four-dimensional supersymmetric theory whose Lagrangian contains moduli-dependent couplings $F_{g}(t)$, where $t$ denotes the Kähler moduli of the Calabi-Yau. When these couplings are expanded in the large radius limit, they are of the form

$$
F_{g}(t)=\sum_{\beta \in H_{2}(X)} N_{g, \beta} \mathrm{e}^{-\beta \cdot t},
$$

where $N_{g, \beta}$ are the Gromov-Witten invariants for the class $\beta$ at genus $g$ (see Sect. 3 below for details on this). It turns out that there is a simplified version of string theory, called topological string theory, which captures precisely the information contained in these couplings. Topological string theory comes in two versions, called the A and the B model (see Hori et al. (2003) and Mariño (2005) for a review). Type A topological string theory is related to GromovWitten theory, and its free energy at genus $g$ is precisely given by (1). Type $\mathrm{B}$ topological string theory is related to the deformation theory of complex structures of the Calabi-Yau manifold. In the last years, various dualities of string theory have led to powerful techniques to compute these couplings, hence Gromov-Witten invariants:

1. Mirror symmetry. Mirror symmetry relates type A theory on a CalabiYau manifold $X$ to type B theory on the mirror manifold $\widetilde{X}$. When the mirror of the Calabi-Yau $X$ is known, this leads to a complete solution at genus zero in terms of variation of the complex structures of $\widetilde{X}$. For genus $g \geq 1$, mirror symmetry can be combined with the holomorphic anomaly equations of Bershadsky et al. (1994) to obtain $F_{g}(t)$. However, this does not provide the full solution to the model due to the so-called holomorphic ambiguity. On the other hand, mirror symmetry and the holomorphic anomaly equation are very general and work for both compact and noncompact Calabi-Yau manifolds.

2. Large $N$ dualities. Large $N$ dualities lead to a computation of the $F_{g}(t)$ couplings in terms of correlation functions and partition functions in Chern-Simons theory. Although this was formulated originally only for the resolved conifold, one ends up with a general theory - the theory of the topological vertex, introduced in Aganagic et al. (2005) - which leads to a complete solution on toric Calabi-Yau manifolds. The theory of the topological vertex is closely related to localization and to Hodge integrals, and it can be formulated in a rigorous mathematical way (see $\mathrm{Li}$ et al. 2004). 
3. Heterotic duality. When the Calabi-Yau manifold has the structure of a K3 fibration, type IIA theory often has a heterotic dual, and the evaluation of $F_{g}(t)$ restricted to the K3 fiber can be reduced to a one-loop integral in heterotic string theory. This leads to explicit, conjectural formulae for Gromov-Witten invariants in terms of modular forms.

In this lectures, I will summarize the approach to Gromov-Witten invariants on toric Calabi-Yau threefolds based on large $N$ dualities. Since the large $N$ duality/topological vertex approach computes Gromov-Witten invariants in terms of Chern-Simons knot and link invariants, Sect. 2 is devoted to a review of these. Section 3 reviews topological strings and Gromov-Witten invariants, and gives some information about the open string case. Section 4 introduces the class of geometries we will deal with, namely toric (noncompact) Calabi-Yau manifolds, and we present a useful graphical way to represent these manifolds which constitutes the geometric core of the theory of the topological vertex. Finally, in Sect.5, we define the vertex and present some explicit formulae for it and some simple applications. A brief Appendix contains useful information about symmetric polynomials.

It has not been possible to present all the relevant background and physical derivations in this set of lectures. However, these topics have been extensively reviewed for example in the book Mariño (2005), to which we refer for further information and/or references.

\section{Chern-Simons Theory}

\subsection{Basic Ingredients}

In a groundbreaking paper, Witten (1989) showed that Chern-Simons gauge theory, which is a quantum field theory in three dimensions, provides a physical description of a wide class of invariants of three-manifolds and of knots and links in three-manifolds. ${ }^{1}$ The Chern-Simons action with gauge group $G$ on a generic three-manifold $M$ is defined by

$$
S=\frac{k}{4 \pi} \int_{M} \operatorname{Tr}\left(A \wedge d A+\frac{2}{3} A \wedge A \wedge A\right) .
$$

Here, $k$ is the coupling constant, and $A$ is a $G$-gauge connection on the trivial bundle over $M$. In the following, we will mostly consider Chern-Simons theory with gauge group $G=U(N)$.

Chern-Simons theory is an example of a topological field theory. The reason is that the Chern-Simons theory action does not involve the metric of $M$ in order to be defined, and the partition function

$$
Z(M)=\int[\mathcal{D} A] \mathrm{e}^{\mathrm{i} S}
$$

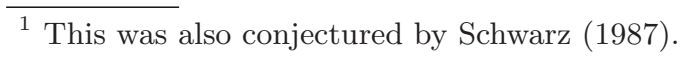


should define a topological invariant of the manifold $M$. The fact, however, that the classical Lagrangian is metric independent is not, in general, sufficient to guarantee that the quantum theory will preserve this invariance, since there could be anomalies in the quantization process that spoil the classical symmetry. A detailed analysis due to Witten (1989) shows that, in the case of Chern-Simons theory, topological invariance is preserved quantum mechanically, but with an extra subtlety: the invariant depends not only on the three-manifold but also on a choice of framing, i.e. a choice of trivialization of the bundle $T M \oplus T M$. The choice of framing changes the value of the partition function in a very precise way: if the framing is changed by $n$ units, the partition function $Z(M)$ changes as follows:

$$
Z(M) \rightarrow \exp \left[\frac{\pi \mathrm{i} n c}{12}\right] Z(M),
$$

where

$$
c=\frac{k d}{k+y} .
$$

In this equation, $d$ and $y$ are, respectively, the dimension and the dual Coxeter number of the group $G$ (for $G=U(N), y=N$ ). As explained by Atiyah (1990), for every three-manifold there is in fact a canonical choice of framing, and the different choices are labelled by an integer $s \in \mathbf{Z}$ in such a way that $s=0$ corresponds to the canonical framing. In the following, unless otherwise stated, all the results for the partition functions of Chern-Simons theory will be presented in the canonical framing.

Besides providing invariants of three-manifolds, Chern-Simons theory also provides invariants of knots and links inside three-manifolds (for a survey of modern knot theory, see Lickorish 1998, and Prasolov and Sossinsky 1997). Some examples of knots and links are depicted in Fig. 1. Given an oriented knot $\mathcal{K}$ in $\mathbf{S}^{3}$, we can consider the trace of the holonomy of the gauge connection around $\mathcal{K}$ in a given irreducible representation $R$ of $U(N)$. This gives the Wilson loop operator:

$$
W_{R}^{\mathcal{K}}(A)=\operatorname{Tr}_{R} U_{\mathcal{K}},
$$

where

$$
U_{\mathcal{K}}=\mathrm{P} \exp \oint_{\mathcal{K}} A
$$

is the holonomy around the knot. The operator in equation (6) is a gaugeinvariant operator whose definition does not involve the metric on the threemanifold, therefore it is an observable of Chern-Simons theory regarded as a topological field theory. The irreducible representations of $U(N)$ will be labelled by highest weights or equivalently by the lengths of rows in a Young 


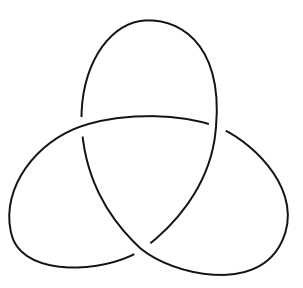

$3_{1}$

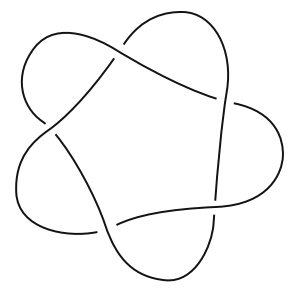

5,

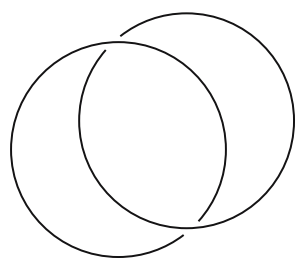

$2_{1}^{2}$

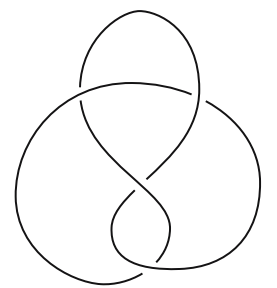

$4_{1}$

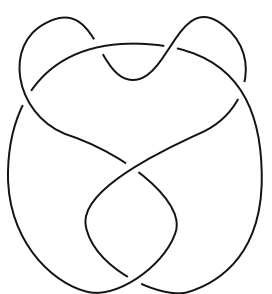

6

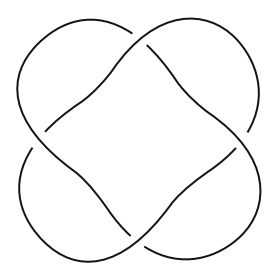

$4_{1}^{2}$

Fig. 1. Some knots and links. In the notation $x_{n}^{L}, x$ indicates the number of crossings, $L$ the number of components (when it is a link with $L>1$ ) and $n$ is a number used to enumerate knots and links in a given set characterized by $x$ and $L$. The knot $3_{1}$ is also known as the trefoil knot, while $4_{1}$ is known as the figure-eight knot. The link $2_{1}^{2}$ is called the Hopf link

tableau, $l_{i}$, where $l_{1} \geq l_{2} \geq \cdots$. If we now consider a link $\mathcal{L}$ with components $\mathcal{K}_{\alpha}, \alpha=1, \cdots, L$, we can in principle compute the normalized correlation function,

$$
W_{R_{1} \cdots R_{L}}(\mathcal{L})=\left\langle W_{R_{1}}^{\mathcal{K}_{1}} \cdots W_{R_{L}}^{\mathcal{K}_{L}}\right\rangle=\frac{1}{Z(M)} \int[\mathcal{D} A]\left(\prod_{\alpha=1}^{L} W_{R_{\alpha}}^{\mathcal{K}_{\alpha}}\right) \mathrm{e}^{i S} .
$$

The unnormalized correlation function will be denoted by $Z_{R_{1} \cdots R_{L}}(\mathcal{L})$. The topological character of the action, and the fact that the Wilson loop operators can be defined without using any metric on the three-manifold, indicate that (8) is a topological invariant of the link $\mathcal{L}$. Similarly to what happens with the partition function, in order to define the invariant of the link we need some extra information due to quantum ambiguities in the correlation function (8). 
For further use we notice that, given two linked oriented knots $\mathcal{K}_{1}, \mathcal{K}_{2}$, one can define an elementary topological invariant, the linking number, by

$$
\operatorname{lk}\left(\mathcal{K}_{1}, \mathcal{K}_{2}\right)=\frac{1}{2} \sum_{\mathrm{p}} \epsilon(p),
$$

where the sum is over all crossing points, and $\epsilon(p)= \pm 1$ is a sign associated to the crossings as indicated in Fig. 2. The linking number of a link $\mathcal{L}$ with components $\mathcal{K}_{\alpha}, \alpha=1, \cdots, L$, is defined by

$$
\operatorname{lk}(\mathcal{L})=\sum_{\alpha<\beta} \operatorname{lk}\left(\mathcal{K}_{\alpha}, \mathcal{K}_{\beta}\right)
$$

For example, once an orientation is chosen for the two components of the Hopf

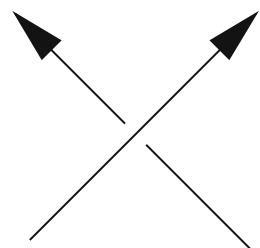

$+1$

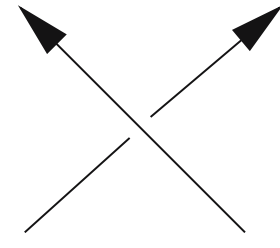

$-1$

Fig. 2. When computing the linking number of two knots, the crossings are assigned a sign \pm 1 as indicated in the figure

link $2_{1}^{2}$ shown in Fig. 1, one finds two inequivalent oriented links with linking numbers \pm 1 .

Some of the correlation functions of Wilson loops in Chern-Simons theory turn out to be closely related to important polynomial invariants of knots and links. For example, one of the most important polynomial invariants of a link $\mathcal{L}$ is the HOMFLY polynomial $P_{\mathcal{L}}(q, \lambda)$, which depends on two variables $q$ and $\lambda$ and was introduced by Freyd et al. (1985). This polynomial turns out to be related to the correlation function (8) when the gauge group is $U(N)$ and all the components are in the fundamental representation $R_{\alpha}=\square$. More precisely, we have

$$
W_{\square \cdots \square}(\mathcal{L})=\lambda^{\mathrm{lk}(\mathcal{L})}\left(\frac{\lambda^{\frac{1}{2}}-\lambda^{-\frac{1}{2}}}{q^{\frac{1}{2}}-q^{-\frac{1}{2}}}\right) P_{\mathcal{L}}(q, \lambda)
$$

where $\operatorname{lk}(\mathcal{L})$ is the linking number of $\mathcal{L}$, and the variables $q$ and $\lambda$ are related to the Chern-Simons variables as

$$
q=\mathrm{e}^{x}, \quad x=\frac{2 \pi \mathrm{i}}{k+N}, \quad \lambda=q^{N} .
$$


When $N=2$ the HOMFLY polynomial reduces to a one-variable polynomial, the Jones polynomial. When the gauge group of Chern-Simons theory is $S O(N), W_{\square \cdots \square}(\mathcal{L})$ is closely related to the Kauffman polynomial. For the mathematical definition and properties of these polynomials, see, for example, Lickorish (1998).

\subsection{Perturbative Approach}

The partition function and correlation functions of Wilson loops in ChernSimons theory can be computed in a variety of ways. We will here present the basic results of Chern-Simons perturbation theory for the partition function. Since our main interest will be the non-perturbative results of Witten (1989), we will be rather sketchy. For more information on Chern-Simons perturbation theory, we refer the reader to Dijkgraaf (1995) and Labastida (1999) for a physical point of view, and Bar-Natan (1995) and Ohtsuki (2002), for a mathematical perspective.

In the computation of the partition function in perturbation theory, we have first to find the classical solutions of the Chern-Simons equations of motion. If we write $A=\sum_{a} A^{a} T_{a}$, where $T_{a}$ is a basis of the Lie algebra, we find

$$
\frac{\delta S}{\delta A_{\mu}^{a}}=\frac{k}{4 \pi} \epsilon^{\mu \nu \rho} F_{\nu \rho}^{a},
$$

therefore the classical solutions are just flat connections on $M$. Flat connections are in one-to-one correspondence with group homomorphisms

$$
\pi_{1}(M) \rightarrow G .
$$

For example, if $M=\mathbf{S}^{3} / \mathbf{Z}_{p}$ is the lens space $L(p, 1)$, one has $\pi_{1}(L(p, 1))=\mathbf{Z}_{p}$, and flat connections are labelled by homomorphisms $\mathbf{Z}_{p} \rightarrow G$. Let us assume that the flat connections on $M$ are a discrete set of points (this happens, for example, if $M$ is a rational homology sphere, since in that case $\pi_{1}(M)$ is a finite group). In that situation, one expresses $Z(M)$ as a sum of terms associated to stationary points:

$$
Z(M)=\sum_{c} Z^{(c)}(M),
$$

where $c$ labels the different flat connections $A^{(c)}$ on $M$. Each of the $Z^{(c)}(M)$ will be an asympotic series in $1 / k$ of the form

$$
Z^{(c)}(M)=Z_{1-\text { loop }}^{(c)}(M) \exp \left\{\sum_{\ell=1}^{\infty} S_{\ell}^{(c)} x^{\ell}\right\} .
$$

In this equation, $x$ is the effective expansion parameter:

$$
x=\frac{2 \pi i}{k+y},
$$


which takes into account a quantum shift $k \rightarrow k+y$ due to finite renormalization effects. The one-loop correction $Z_{1-\text { loop }}^{(c)}(M)$ was first analyzed by Witten (1989), and has been studied in great detail since then (Freed and Gompf 1991; Jeffrey 1992; Rozansky 1995). It has the form

$$
Z_{1-\text { loop }}^{(c)}(M)=\frac{(2 \pi x)^{\frac{1}{2}\left(\operatorname{dim} H_{c}^{0}-\operatorname{dim} H_{c}^{1}\right)}}{\operatorname{vol}\left(H_{c}\right)} \mathrm{e}^{-\frac{1}{x} S_{\mathrm{CS}}\left(A^{(c)}\right)-\frac{i \pi}{4} \varphi} \sqrt{\left|\tau_{R}^{(c)}\right|},
$$

where $H_{c}^{0,1}$ are the cohomology groups with values in the Lie algebra of $G$ associated to the flat connection $A^{(c)}, \tau_{R}^{(c)}$ is the Reidemeister-Ray-Singer torsion of $A^{(c)}, H_{c}$ is the isotropy group of $A^{(c)}$, and $\varphi$ is a certain phase. Notice that, for the trivial flat connection $A^{(c)}=0, H_{c}=G$.

Let us focus on the terms in (15) corresponding to the trivial connection, which will be denoted by $S_{\ell}$. Diagramatically, the free energy is computed by connected bubble diagrams made out of trivalent vertices (since the interaction in the Chern-Simons action is cubic). We will refer to these diagrams as connected trivalent graphs. $S_{\ell}$ is the contribution of connected trivalent graphs with $2 \ell$ vertices and $\ell+1$ loops. For each of these graphs we have to compute a group factor and a Feynman integral. However, not all these graphs are independent, since the underlying Lie algebra structure imposes the Jacobi identity:

$$
\sum_{e}\left(f_{a b e} f_{e d c}+f_{d a e} f_{e b c}+f_{a c e} f_{e d b}\right)=0
$$

This leads to the graph relation known as the IHX relation. Also, antisymmetry of $f_{a b c}$ leads to the so-called AS relation (see, for example, Bar-Natan 1995; Dijkgraaf 1995; Ohtsuki 2002). The existence of these relations suggests to define an equivalence relation in the space of connected trivalent graphs by quotienting by the IHX and the AS relations, and this gives the so-called graph homology. The space of homology classes of connected diagrams will be denoted by $\mathcal{A}(\emptyset)^{\text {conn }}$. This space is graded by half the number of vertices $\ell$, and this number gives the degree of the graph. The space of homology classes of graphs at degree $\ell$ is then denoted by $\mathcal{A}(\emptyset)_{\ell}^{\text {conn }}$. For every $\ell$, this is a finite-dimensional vector space of dimension $d(\ell)$. The dimensions of these spaces are explicitly known for low degrees, see, for example, Bar-Natan (1995), and we have listed some of them in Table 1. Given any group $G$, we have a map

$$
r_{G}: \mathcal{A}(\emptyset)^{\operatorname{conn}} \longrightarrow \mathbf{R}
$$

that associates to every graph $\Gamma$ its group theory factor $r_{G}(\Gamma)$. This map is of course well defined, since different graphs in the same homology class $\mathcal{A}(\emptyset)^{\text {conn }}$ lead by definition to the same group factor. This map is an example of a weight system for $\mathcal{A}(\emptyset)^{\text {conn }}$. Every gauge group gives a weight system for $\mathcal{A}(\emptyset)^{\text {conn }}$, but one may, in principle, find weight systems not associated to 
gauge groups, although so far the only known example is the one constructed by Rozansky and Witten (1997), which instead uses hyperKähler manifolds. We can now state very precisely what is the structure of the $S_{\ell}$ appearing in (15): since the Feynman diagrams can be grouped into homology classes, we have

Table 1. Dimensions $d(\ell)$ of $\mathcal{A}(\emptyset)_{\ell}^{\text {conn }}$ up to $\ell=10$

\begin{tabular}{|c|c|c|c|c|c|c|c|c|c|c|}
\hline$\ell$ & 1 & 2 & 3 & 4 & 5 & 6 & 7 & 8 & 9 & 10 \\
\hline$d(\ell)$ & 1 & 1 & 1 & 2 & 2 & 3 & 4 & 5 & 6 & 8 \\
\hline
\end{tabular}

$$
S_{\ell}=\sum_{\Gamma \in \mathcal{A}(\emptyset)_{\ell}^{\text {conn }}} r_{G}(\Gamma) I_{\Gamma}(M)
$$

The factors $I_{\Gamma}(M)$ appearing in (20) are certain sums of integrals of propagators over $M$. It was shown by Axelrod and Singer (1992) that these are differentiable invariants of the three-manifold $M$, and since the dependence on the gauge group has been factored out, they only capture topological information of $M$, in contrast to $Z(M)$, which also depends on the choice of the gauge group. These are the universal perturbative invariants defined by Chern-Simons theory. Notice that, at every order $\ell$ in perturbation theory, there are $d(\ell)$ independent perturbative invariants. Of course, these invariants inherit from $\mathcal{A}(\emptyset)_{\ell}^{\text {conn }}$ the structure of a finite-dimensional vector space, and in particular one can choose a basis of trivalent graphs. A possible choice for $\ell \leq 5$ is the following (Sawon 2004):

$$
\begin{aligned}
& \ell=1: \\
& \ell=2: \\
& \ell=3: \\
& \ell=4: \\
& \ell=5: \quad .
\end{aligned}
$$

We will denote the graphs with $k$ circles joined by lines by $\theta_{k}$. Therefore, the graph corresponding to $\ell=1$ will be denoted by $\theta$, the graph corresponding to $\ell=2$ will be denoted $\theta_{2}$, and so on.

Notice that Chern-Simons theory detects the graph homology through the weight system associated to Lie algebras, so in principle it could happen that there is an element of graph homology that is not detected by these weight systems. There is, however, a very elegant mathematical definition of the universal perturbative invariant of a three-manifold that works directly in the graph homology. This is called the LMO invariant (Le et al. 1998) and it is a formal linear combination of homology graphs with rational coefficients: 


$$
\omega(M)=\sum_{\Gamma \in \mathcal{A}(\emptyset)^{\text {conn }}} I_{\Gamma}^{\mathrm{LMO}}(M) \Gamma \in \mathcal{A}(\emptyset)^{\mathrm{conn}}[\mathbf{Q}] .
$$

It is believed that the universal invariants extracted from Chern-Simons perturbation theory agree with the LMO invariant. More precisely, since the LMO invariant $\omega(M)$ is taken to be 0 for $\mathbf{S}^{3}$, we have:

$$
I_{\Gamma}^{\mathrm{LMO}}(M)=I_{\Gamma}(M)-I_{\Gamma}\left(\mathbf{S}^{3}\right),
$$

as long as the graph $\Gamma$ is detected by Lie algebra weight systems. In that sense the LMO invariant is more refined than the universal perturbative invariants extracted from Chern-Simons theory; see Ohtsuki (2002) for a detailed introduction to the LMO invariant and its properties.

The computation of $S_{\ell}$ involves the evaluation of group factors of Feynman diagrams, which we have denoted by $r_{G}(\Gamma)$ above. Here, we give some details about how to evaluate these factors when $G=U(N)$, following the diagrammatic techniques of Cvitanovic (1976) and Bar-Natan (1995). A systematic discussion of these techniques can be found in Cvitanovic (2004).

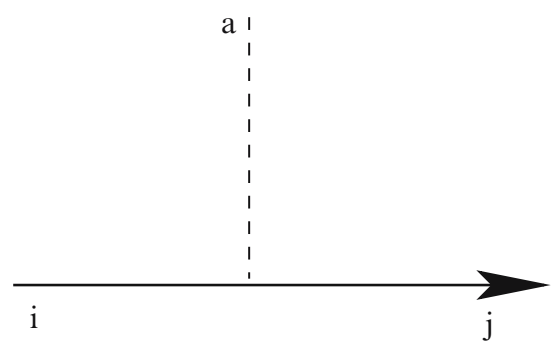

Fig. 3. Graphic representation of the generator $\left(T_{a}\right)_{i j}$ of a Lie algebra

The basic idea to evaluate group factors is very similar to the double-line notation of 't Hooft (1974), and it amounts to expressing indices in the adjoint representation in terms of indices in the fundamental (and anti-fundamental) representation. The resulting diagrams are often called fatgraphs. In the case of $U(N)$, the adjoint representation is just the tensor product of the fundamental and the anti-fundamental representation. Let us first normalize the trace in the fundamental representation by setting

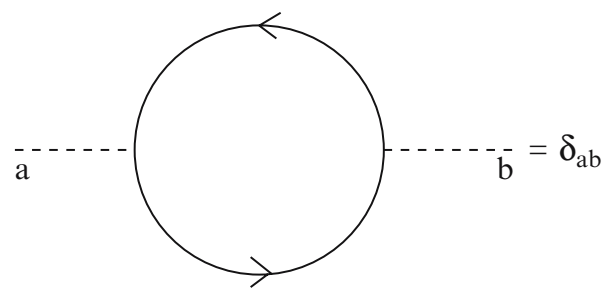

Fig. 4. Graphic representation of the normalization condition (24) 


$$
\operatorname{Tr}\left(T_{a} T_{b}\right)=\delta_{a b}, \quad a, b=1, \cdots, N^{2} .
$$

One can then see that

$$
\sum_{a}\left(T_{a}\right)_{i j}\left(T_{a}\right)_{k l}=\delta_{i l} \delta_{k j}
$$

If we represent the generator $\left(T_{a}\right)_{i j}$ as in Fig. 3, the relation (25) can in turn be represented as Fig. 5. This is simply the statement that the adjoint representation of $U(N)$ is given by $V_{N} \otimes V_{N}^{*}$. Similarly, the normalization condition (24) is graphically represented as Fig. 4. The evaluation of group

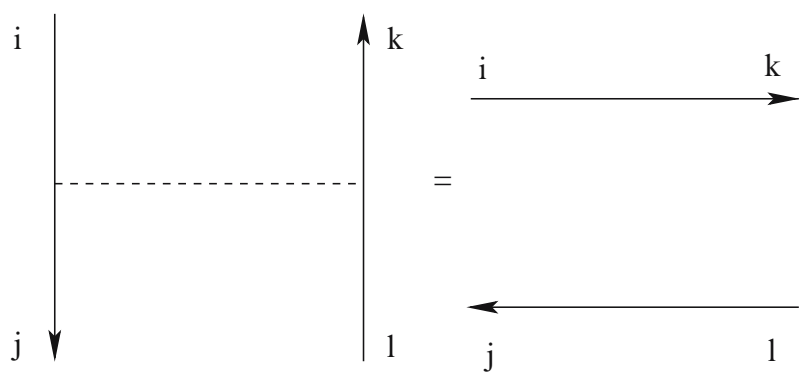

Fig. 5. Graphic representation of (25)

factors of Feynman diagrams involves, of course, the structure constants of the Lie algebra $f_{a b c}$, associated to the cubic vertex. By tracing the defining relation of the structure constants we find

$$
f_{a b c}=\operatorname{Tr}\left(T_{a} T_{b} T_{c}\right)-\operatorname{Tr}\left(T_{b} T_{a} T_{c}\right),
$$

which we represent as Fig. 6. Putting this together with Fig. 5, we obtain the

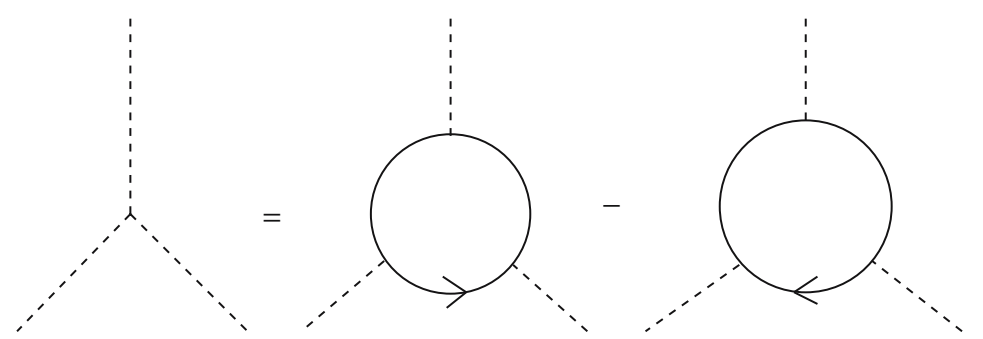

Fig. 6. Graphic representation of the relation (26) between structure constants and generators 
graphical rule represented in Fig. 7. We can interpret this as a rule that tells us how to split a single-line Feynman diagram of the $U(N)$ theory into fatgraphs: given a Feynman diagram, we substitute each vertex by the double line vertex without twists, minus the double-line vertex with twists in all edges. If the diagram has $2 \ell$ vertices, we will generate $4^{\ell}$ fatgraphs (some of them may be equal), with a \pm sign, which can be interpreted as Riemann surfaces with holes. The group factor of a fatgraph with $h$ holes is simply $N^{h}$.

Example. As an example of the above procedure, One can use the above rules to compute the group factor of the two-loop Feynman diagram

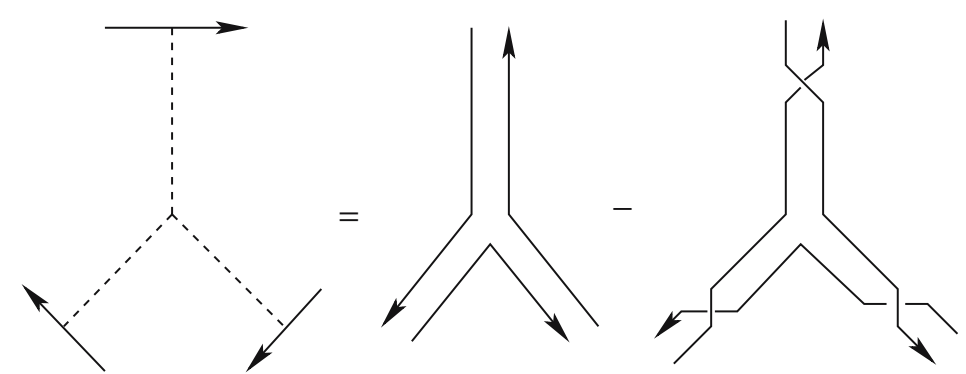

Fig. 7. Graphic rule to transform Feynman diagrams into double-line diagrams

By resolving the two vertices we obtain two different fatgraphs: the graph in Fig. 8 with weight 2, and the graph in Fig. 9 with weight -2 . One then finds:

$$
r_{U(N)}(\theta)=2 N\left(N^{2}-1\right) .
$$

Similarly, the same procedure gives

$$
r_{U(N)}\left(\theta_{2}\right)=4 N^{2}\left(N^{2}-1\right) .
$$

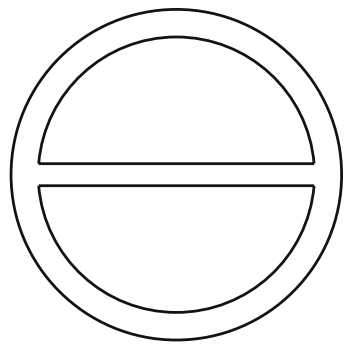

Fig. 8. A fatgraph obtained from the Feynman diagram (27) 


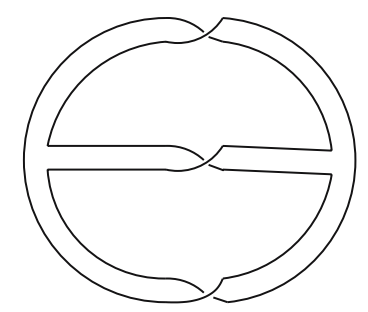

Fig. 9. Another fatgraph obtained from (27)

It is easy to see from the evaluation of group factors that the perturbative expansion of the free energy of Chern-Simons theory around the trivial connection can be written in the form

$$
F=\sum_{g=0}^{\infty} \sum_{h=1}^{\infty} F_{g, h} x^{2 g-2+h} N^{h} .
$$

In fact, this structure for the partition function holds for any quantum theory containing only fields in the adjoint representation ('t Hooft 1974). One can also reorganize the perturbative series (30) as

$$
F=\sum_{g=0}^{\infty} F_{g}(t) g_{s}^{2 g-2},
$$

where $t$ is called the 't Hooft coupling of Chern-Simons theory and it is given by

$$
t=N x
$$

and $F_{g}(t)$ is defined by summing over all holes keeping the genus $g$ fixed:

$$
F_{g}(t)=\sum_{h=1}^{\infty} F_{g, h} t^{h} .
$$

We will see later in this section how to compute the coefficients $F_{g, h}$ and the function $F_{g}(t)$ for Chern-Simons theory on $\mathbf{S}^{3}$.

\subsection{Non-Perturbative Solution}

As was shown by Witten (1989), Chern-Simons theory is exactly solvable by using non-perturbative methods and the relation to the Wess-Zumino-Witten (WZW) model. In order to present this solution, it is convenient to recall some basic facts about the canonical quantization of the model.

Let $M$ be a three-manifold with boundary given by a Riemann surface $\Sigma$. We can insert a general operator $\mathcal{O}$ in $M$, which will, in general, be a 
product of Wilson loops along different knots and in arbitrary representations of the gauge group. We will consider the case in which the Wilson loops do not intersect the surface $\Sigma$. The path integral over the three-manifold with boundary $M$ gives a wavefunction $\Psi_{M, \mathcal{O}}(\mathcal{A})$ that is a functional of the values of the field on $\Sigma$. Schematically, we have:

$$
\Psi_{M, \mathcal{O}}(\mathcal{A})=\left\langle\mathcal{A} \mid \Psi_{M, \mathcal{O}}\right\rangle=\int_{\left.A\right|_{\Sigma}=\mathcal{A}} \mathcal{D} A \mathrm{e}^{\mathrm{i} S} \mathcal{O} .
$$

In fact, associated to the Riemann surface $\Sigma$ we have a Hilbert space $\mathcal{H}(\Sigma)$, which can be obtained by doing canonical quantization of Chern-Simons theory on $\Sigma \times \mathbf{R}$. Before spelling out in detail the structure of these Hilbert spaces, let us make some general considerations about the computation of physical quantities.

In the context of canonical quantization, the partition function can be computed as follows. We first perform a Heegaard splitting of the three-manifold, i.e. we represent it as the connected sum of two three-manifolds $M_{1}$ and $M_{2}$ sharing a common boundary $\Sigma$, where $\Sigma$ is a Riemann surface. If $f: \Sigma \rightarrow \Sigma$ is a homeomorphism, we will write $M=M_{1} \cup_{f} M_{2}$, so that $M$ is obtained by gluing $M_{1}$ to $M_{2}$ through their common boundary and using the homeomorphism $f$. This is represented in Fig. 10. We can then compute the full path integral (3) over $M$ by computing first the path integral over $M_{1}$ to obtain a state $\left|\Psi_{M_{1}}\right\rangle$ in $\mathcal{H}(\Sigma)$. The boundary of $M_{2}$ is also $\Sigma$, but with opposite orientation, so its Hilbert space is the dual space $\mathcal{H}^{*}(\Sigma)$. The path integral over $M_{2}$ then produces a state $\left\langle\Psi_{M_{2}}\right| \in \mathcal{H}^{*}(\Sigma)$. The homeomorphism $f: \Sigma \rightarrow \Sigma$ will be represented by an operator acting on $\mathcal{H}(\Sigma)$,

$$
U_{f}: \mathcal{H}(\Sigma) \rightarrow \mathcal{H}(\Sigma) .
$$

and the partition function can be finally evaluated as

$$
Z(M)=\left\langle\Psi_{M_{2}}\left|U_{f}\right| \Psi_{M_{1}}\right\rangle .
$$

Therefore, if we know explicitly what the wavefunctions and the operators associated to homeomorphisms are, we can compute the partition function. The result of the computation is, of course, independent of the particular Heegaard splitting of $M$.

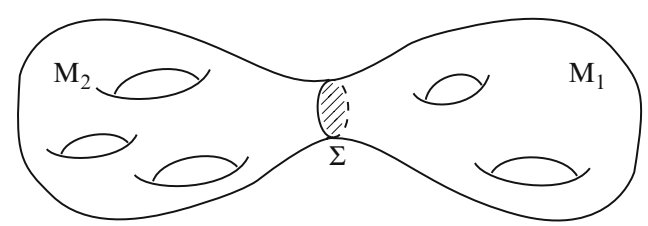

Fig. 10. Heegaard splitting of a three-manifold $M$ into two three-manifolds $M_{1}$ and $M_{2}$ with a common boundary $\Sigma$ 
One of the most fundamental results of Witten (1989) is a precise description of $\mathcal{H}(\Sigma)$ : it is the space of conformal blocks of a WZW model on $\Sigma$ with gauge group $G$ and level $k$ (for an extensive review of the WZW model, see, for example, Di Francesco et al. 1997). In particular, $\mathcal{H}(\Sigma)$ has finite dimension. We will not review here the derivation of this fundamental result. Instead we will use the relevant information from the WZW model in order to solve Chern-Simons theory in some important cases.

The description of the space of conformal blocks on Riemann surfaces can be made very explicit when $\Sigma$ is a sphere or a torus. For $\Sigma=\mathbf{S}^{2}$, the space of conformal blocks is one-dimensional, so $\mathcal{H}\left(\mathbf{S}^{2}\right)$ is spanned by a single element. For $\Sigma=\mathbf{T}^{2}$, the space of conformal blocks is in one-to-one correspondence with the integrable representations of the affine Lie algebra associated to $G$ at level $k$. We will use the following notations: the fundamental weights of $G$ will be denoted by $\lambda_{i}$, and the simple roots by $\alpha_{i}, i=1, \cdots, r$, where $r$ denotes the rank of $G$. The weight and root lattices of $G$ are denoted by $\Lambda^{w}$ and $\Lambda^{r}$, respectively, and $\left|\Delta_{+}\right|$denotes the number of positive roots. The fundamental chamber $\mathcal{F}_{l}$ is given by $\Lambda^{w} / l \Lambda^{r}$, modded out by the action of the Weyl group. For example, in $S U(N)$ a weight $p=\sum_{i=1}^{r} p_{i} \lambda_{i}$ is in $\mathcal{F}_{l}$ if

$$
\sum_{i=1}^{r} p_{i}<l, \quad \text { and } p_{i}>0, i=1, \cdots, r .
$$

We recall that a representation given by a highest weight $\Lambda$ is integrable if $\rho+\Lambda$ is in the fundamental chamber $\mathcal{F}_{l}$, where $l=k+y$ ( $\rho$ denotes as usual the Weyl vector, given by the sum of the fundamental weights). In the following, the states in the Hilbert state of the torus $\mathcal{H}\left(\mathbf{T}^{2}\right)$ will be denoted by $|p\rangle=|\rho+\Lambda\rangle$ where $\rho+\Lambda \in \mathcal{F}_{l}$, as we have stated, is an integrable representation of the WZW model at level $k$. We will also denote these states by $|R\rangle$, where $R$ is the representation associated to $\Lambda$. The state $|\rho\rangle$ will be denoted by $|0\rangle$. The states $|R\rangle$ can be chosen to be orthonormal (Witten 1989; Elitzur et al. 1989; Labastida and Ramallo 1989), so we have

$$
\left\langle R \mid R^{\prime}\right\rangle=\delta_{R R^{\prime}} .
$$

There is a special class of homeomorphisms of $\mathbf{T}^{2}$ that have a simple expression as operators in $\mathcal{H}\left(\mathbf{T}^{2}\right)$; these are the $\operatorname{SL}(2, \mathbf{Z})$ transformations. Recall that the group $\operatorname{SL}(2, \mathbf{Z})$ consists of $2 \times 2$ matrices with integer entries and unit determinant. If $(1,0)$ and $(0,1)$ denote the two one-cycles of $\mathbf{T}^{2}$, we can specify the action of an $\mathrm{SL}(2, \mathbf{Z})$ transformation on the torus by giving its action on this homology basis. The $\mathrm{SL}(2, \mathbf{Z})$ group is generated by the transformations $T$ and $S$, which are given by

$$
T=\left(\begin{array}{ll}
1 & 1 \\
0 & 1
\end{array}\right), \quad S=\left(\begin{array}{cc}
0 & -1 \\
1 & 0
\end{array}\right) .
$$

Notice that the $S$ transformation exchanges the one-cycles of the torus. These transformations can be lifted to $\mathcal{H}\left(\mathbf{T}^{2}\right)$, and they have the following matrix elements in the basis of integrable representations: 


$$
\begin{aligned}
T_{p p^{\prime}} & =\delta_{p, p^{\prime}} \mathrm{e}^{2 \pi \mathrm{i}\left(h_{p}-c / 24\right)} \\
S_{p p^{\prime}} & =\frac{\mathrm{i}^{\left|\Delta_{+}\right|}}{(k+y)^{r / 2}}\left(\frac{\operatorname{Vol} \Lambda^{w}}{\operatorname{Vol} \Lambda^{r}}\right)^{\frac{1}{2}} \sum_{w \in \mathcal{W}} \epsilon(w) \exp \left(-\frac{2 \pi \mathrm{i}}{k+y} p \cdot w\left(p^{\prime}\right)\right) .
\end{aligned}
$$

In the first equation, $c$ is the central charge of the WZW model, and $h_{p}$ is the conformal weight of the primary field associated to $p$ :

$$
h_{p}=\frac{p^{2}-\rho^{2}}{2(k+y)},
$$

where we recall that $p$ is of the form $\rho+\Lambda$. In the second equation, the sum over $w$ is a sum over the elements of the Weyl group $\mathcal{W}, \epsilon(w)$ is the signature of the element $w$, and $\operatorname{Vol} \Lambda^{w}\left(\operatorname{Vol} \Lambda^{r}\right)$ denote, respectively, the volume of the weight (root) lattice. We will often write $S_{R R^{\prime}}$ for $S_{p p^{\prime}}$, where $p=\rho+\Lambda, p^{\prime}=\rho+\Lambda^{\prime}$ and $\Lambda, \Lambda^{\prime}$ are the highest weights corresponding to the representations $R, R^{\prime}$.

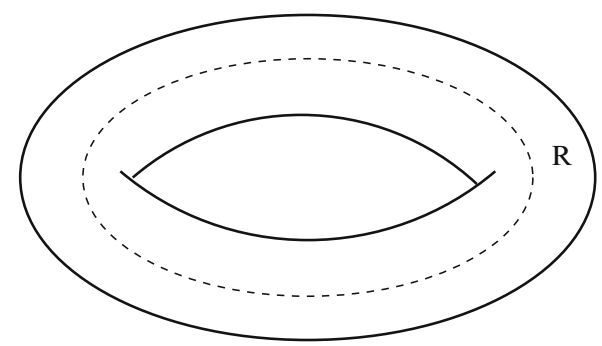

Fig. 11. Performing the path integral on a solid torus with a Wilson line in representation $R$ gives the state $|R\rangle$ in $\mathcal{H}\left(\mathbf{T}^{2}\right)$

What is the description of the states $|R\rangle$ in $\mathcal{H}\left(\mathbf{T}^{2}\right)$ from the point of view of canonical quantization? Consider the solid torus $\mathcal{T}=D \times \mathbf{S}^{1}$, where $D$ is a disc in $\mathbf{R}^{2}$. This is a three-manifold whose boundary is a $\mathbf{T}^{2}$, and it has a non-contractible cycle given by the $\mathbf{S}^{1}$. Let us now consider the Chern-Simons path integral on the solid torus, with the insertion of the operator $\mathcal{O}_{R}=\operatorname{Tr}_{R} U$ given by a Wilson loop in the representation $R$ around the non-contractible cycle, as shown in Fig. 11. In this way, one obtains a state in $\mathcal{H}\left(\mathbf{T}^{2}\right)$, and one has

$$
\left|\Psi_{\mathcal{T}, \mathcal{O}_{R}}\right\rangle=|R\rangle
$$

In particular, the path integral over the solid torus with no operator insertion gives $|0\rangle$, the 'vacuum' state.

These results allow us to compute the partition function of any threemanifold that admits a Heegaard splitting along a torus. Imagine, for example, that we take two solid tori and we glue them along their boundary with the 
identity map. Since a solid torus is a disc times a circle, $D \times \mathbf{S}^{1}$, by performing this operation we get a manifold that is $\mathbf{S}^{1}$ times the two discs glued together along their boundaries. Therefore, with this surgery we obtain $\mathbf{S}^{2} \times \mathbf{S}^{1}$, and (36) then gives

$$
Z\left(\mathbf{S}^{2} \times \mathbf{S}^{1}\right)=\langle 0 \mid 0\rangle=1 .
$$

If we do the gluing, however, after performing an $S$-transformation on the $\mathbf{T}^{2}$ the resulting manifold is instead $\mathbf{S}^{3}$. To see this, notice that the complement to a solid torus inside $\mathbf{S}^{3}$ is indeed another solid torus whose non-contractible cycle is homologous to the contractible cycle in the first torus. We then find

$$
Z\left(\mathbf{S}^{3}\right)=\langle 0|S| 0\rangle=S_{00} .
$$

By using Weyl's denominator formula,

$$
\sum_{w \in \mathcal{W}} \epsilon(w) \mathrm{e}^{w(\rho)}=\prod_{\alpha>0} 2 \sinh \frac{\alpha}{2},
$$

where $\alpha>0$ are positive roots, one finds

$$
Z\left(\mathbf{S}^{3}\right)=\frac{1}{(k+y)^{r / 2}}\left(\frac{\operatorname{Vol} \Lambda^{w}}{\operatorname{Vol} \Lambda^{r}}\right)^{\frac{1}{2}} \prod_{\alpha>0} 2 \sin \left(\frac{\pi(\alpha \cdot \rho)}{k+y}\right) .
$$

The above result can be generalized in order to compute path integrals in $\mathbf{S}^{3}$ with some knots and links. Consider a solid torus where a Wilson line in representation $R$ has been inserted. The corresponding state is $|R\rangle$, as we explained before. If we now glue this to an empty solid torus after an $S$ transformation, we obtain a trivial knot, or unknot, in $\mathbf{S}^{3}$. The path integral with the insertion is then,

$$
Z_{R}=\langle 0|S| R\rangle .
$$

It follows that the normalized vacuum expectation value for the unknot in $\mathbf{S}^{3}$, in representation $R$, is given by

$$
W_{R}(\text { unknot })=\frac{S_{0 R}}{S_{00}}=\frac{\sum_{w \in \mathcal{W}} \epsilon(w) \mathrm{e}^{-\frac{2 \pi \mathrm{i}}{k+y} \rho \cdot w(\Lambda+\rho)}}{\sum_{w \in \mathcal{W}} \epsilon(w) \mathrm{e}^{-\frac{2 \pi \mathrm{i}}{k+y} \rho \cdot w(\rho)}} .
$$

This expression can be written in terms of characters of the group $G$. Remember that the character of the representation $R$, evaluated on an element $a \in \Lambda^{w} \otimes \mathbf{R}$ is defined by

$$
\operatorname{ch}_{R}(a)=\sum_{\mu \in M_{R}} \mathrm{e}^{a \cdot \mu},
$$

where $M_{R}$ is the set of weights associated to the irreducible representation $R$. By using Weyl's character formula we can write 


$$
W_{R}(\text { unknot })=\operatorname{ch}_{R}\left[-\frac{2 \pi \mathrm{i}}{k+y} \rho\right]
$$

Moreover, using (45), we finally obtain

$$
W_{R}(\text { unknot })=\prod_{\alpha>0} \frac{\sin \left(\frac{\pi}{k+y} \alpha \cdot(\Lambda+\rho)\right)}{\sin \left(\frac{\pi}{k+y} \alpha \cdot \rho\right)} .
$$

This quantity is often called the quantum dimension of $R$, and it is denoted by $\operatorname{dim}_{q} R$.

We can also consider a solid torus with a Wilson loop in representation $R$, glued to another solid torus with the representation $R^{\prime}$ through an $S$ transformation. What we obtain is clearly a link in $\mathbf{S}^{3}$ with two components, which is the Hopf link shown in Fig. 1. Carefully taking into account the orientation, we find that this is the Hopf link with linking number +1 . The path integral with this insertion is:

$$
Z_{R R^{\prime}}=\left\langle R^{\prime}|S| R\right\rangle,
$$

so the normalized vacuum expectation value is

$$
\mathcal{W}_{R R^{\prime}} \equiv W_{R R^{\prime}}\left(\operatorname{Hopf}^{+1}\right)=\frac{S_{\bar{R}^{\prime} R}}{S_{00}}=\frac{S_{R^{\prime} R}^{-1}}{S_{00}},
$$

where the superscript +1 refers to the linking number. Here, we have used that the bras $\langle R|$ are canonically associated to conjugate representations $\bar{R}$, and that $S_{\bar{R}^{\prime} R}=S_{R^{\prime} R}^{-1}$ (see for example Di Francesco et al. 1997). Therefore, the Chern-Simons invariant of the Hopf link is essentially an $S$-matrix element. In order to obtain the invariant of the Hopf link with linking number -1, we notice that the two Hopf links can be related by changing the orientation of one of the components. Since this is equivalent to conjugating the representation, we find

$$
W_{R R^{\prime}}\left(\operatorname{Hopf}^{-1}\right)=\frac{S_{R^{\prime} R}}{S_{00}} .
$$

When we take $G=U(N)$, the above vacuum expectation values for unknots and Hopf links can be evaluated very explicitly in terms of Schur polynomials. It is well known that the character of the unitary group in the representation $R$ is given by the Schur polynomial $s_{R}$ (see for example Fulton and Harris 1991). There is a precise relation between the element $a$ on which one evaluates the character in (49) and the variables entering the Schur polynomial. Let $\mu_{i}$, $i=1, \cdots, N$, be the weights associated to the fundamental representation of $U(N)$. Notice that, if $R$ is given by a Young tableau whose rows have lengths $l_{1} \geq \cdots \geq l_{N}$, then $\Lambda_{R}=\sum_{i} l_{i} \mu_{i}$. We also have

$$
\rho=\sum_{i=1}^{N} \frac{1}{2}(N-2 i+1) \mu_{i} .
$$


Let $a \in \Lambda^{w} \otimes \mathbf{R}$ be given by

$$
a=\sum_{i=1}^{N} a_{i} \mu_{i}
$$

Then,

$$
\operatorname{ch}_{R}[a]=s_{R}\left(x_{i}=\mathrm{e}^{a_{i}}\right) .
$$

For example, in the case of the quantum dimension, one has $\operatorname{dim}_{q} R=\operatorname{dim}_{q} \bar{R}$, and we find

$$
\operatorname{dim}_{q} R=s_{R}\left(x_{i}=q^{\frac{1}{2}(N-2 i+1)}\right),
$$

where $q$ is given in (12). By using that $s_{R}$ is homogeneous of degree $\ell(R)$ in the variables $x_{i}$ we finally obtain

$$
\operatorname{dim}_{q} R=\lambda^{\ell(R) / 2} s_{R}\left(x_{i}=q^{-i+\frac{1}{2}}\right)
$$

where $\lambda=q^{N}$ as in (12), and there are $N$ variables $x_{i}$. The quantum dimension can be written very explicitly in terms of the $q$-numbers:

$$
[a]=q^{\frac{a}{2}}-q^{-\frac{a}{2}}, \quad[a]_{\lambda}=\lambda^{\frac{1}{2}} q^{\frac{a}{2}}-\lambda^{-\frac{1}{2}} q^{-\frac{a}{2}} .
$$

If $R$ corresponds to a Young tableau with $c_{R}$ rows of lengths $l_{i}, i=1, \cdots, c_{R}$, the quantum dimension is given by:

$$
\operatorname{dim}_{q} R=\prod_{1 \leq i<j \leq c_{R}} \frac{\left[l_{i}-l_{j}+j-i\right]}{[j-i]} \prod_{i=1}^{c_{R}} \frac{\prod_{v=-i+1}^{l_{i}-i}[v]_{\lambda}}{\prod_{v=1}^{l_{i}}\left[v-i+c_{R}\right]}
$$

It is easy to check that in the limit $k+N \rightarrow \infty$ (i.e. in the semi-classical limit) the quantum dimension becomes the dimension of the representation $R$. Notice that the quantum dimension is a rational function of $q^{ \pm \frac{1}{2}}, \lambda^{ \pm \frac{1}{2}}$. This is a general property of all normalized vacuum expectation values of knots and links in $\mathbf{S}^{3}$.

The $S$-matrix elements that appear in (53) and (54) can be evaluated through the explicit expression (40), by using the relation between $U(N)$ characters and Schur functions that we explained above. Notice first that

$$
\frac{S_{R_{1} R_{2}}^{-1}}{S_{00}}=\operatorname{ch}_{R_{1}}\left[\frac{2 \pi \mathrm{i}}{k+y}\left(\Lambda_{R_{2}}+\rho\right)\right] \operatorname{ch}_{R_{2}}\left[\frac{2 \pi \mathrm{i}}{k+y} \rho\right] .
$$

If we denote by $l_{i}^{R_{2}}, i=1, \cdots, c_{R_{2}}$ the lengths of rows for the Young tableau corresponding to $R_{2}$, it is easy to see that

$$
\mathcal{W}_{R_{1} R_{2}}(q, \lambda)=(\lambda q)^{\frac{\ell\left(R_{1}\right)}{2}} s_{R_{1}}\left(x_{i}=q^{l_{i}^{R_{2}}-i}\right) \operatorname{dim}_{q} R_{2},
$$


where we set $l_{i}^{R_{2}}=0$ for $i>c_{R_{2}}$. A convenient way to evaluate $s_{R_{1}}\left(x_{i}=\right.$ $q^{l_{i}^{R}-i}$ ) for a partition $\left\{l_{i}^{R}\right\}_{\left\{i=1, \cdots, c_{R}\right\}}$ associated to $R$ is to use the Jacobi-Trudi formula (188). It is easy to show that the generating functional of elementary symmetric functions (184) for this specialization is given by

$$
E_{R}(t)=E_{\emptyset}(t) \prod_{j=1}^{c_{R}} \frac{1+q^{l_{j}^{R}-j} t}{1+q^{-j} t}
$$

where

$$
E_{\emptyset}(t)=1+\sum_{n=1}^{\infty} a_{n} t^{n}
$$

and the coefficients $a_{n}$ are defined by

$$
a_{n}=\prod_{r=1}^{n} \frac{1-\lambda^{-1} q^{r-1}}{q^{r}-1} .
$$

The formula (62), together with the expressions above for $E_{R}(t)$, provides an explicit expression for (53) as a rational function of $q^{ \pm \frac{1}{2}}, \lambda^{ \pm \frac{1}{2}}$, and it was first written down by Morton and Lukac (2003).

\subsection{Framing Dependence}

In the above discussion on the correlation functions of Wilson loops we have glossed over an important ingredient. We already mentioned that, in order to define the partition function of Chern-Simons theory at the quantum level, one has to specify a framing of the three-manifold. It turns out that the evaluation of correlation functions like (8) also involves a choice of framing of the knots, as discovered by Witten (1989).

A good starting point to understand the framing is to take Chern-Simons theory with gauge group $U(1)$. The Abelian Chern-Simons theory turns out to be extremely simple, since the cubic term in (2) drops out, and we are left with a Gaussian theory (Polyakov 1988). U(1) representations are labelled by integers, and the correlation function (8) can be computed exactly. In order to do that, however, one has to choose a framing for each of the knots $\mathcal{K}_{\alpha}$. This arises as follows: in evaluating the correlation function, contractions of the holonomies corresponding to different $\mathcal{K}_{\alpha}$ produce the following integral:

$$
\operatorname{lk}\left(\mathcal{K}_{\alpha}, \mathcal{K}_{\beta}\right)=\frac{1}{4 \pi} \oint_{\mathcal{K}_{\alpha}} \mathrm{d} x^{\mu} \oint_{\mathcal{K}_{\beta}} \mathrm{d} y^{\nu} \epsilon_{\mu \nu \rho} \frac{(x-y)^{\rho}}{|x-y|^{3}} .
$$

This is a topological invariant, i.e. it is invariant under deformations of the knots $\mathcal{K}_{\alpha}, \mathcal{K}_{\beta}$, and it is, in fact, the Gauss integral representation of their linking number $\operatorname{lk}\left(\mathcal{K}_{\alpha}, \mathcal{K}_{\beta}\right)$ defined in (9). On the other hand, contractions of the holonomies corresponding to the same knot $\mathcal{K}$ involve the integral 


$$
\phi(\mathcal{K})=\frac{1}{4 \pi} \oint_{\mathcal{K}} \mathrm{d} x^{\mu} \oint_{\mathcal{K}} \mathrm{d} y^{\nu} \epsilon_{\mu \nu \rho} \frac{(x-y)^{\rho}}{|x-y|^{3}} .
$$

This integral is well defined and finite (see, for example, Guadagnini et al. 1990), and it is called the cotorsion or writhe of $\mathcal{K}$. It gives the self-linking number of $\mathcal{K}$ : if we project $\mathcal{K}$ on a plane, and we denote by $n_{ \pm}(\mathcal{K})$ the number of positive (negative) crossings as indicated in Fig. 2, then we have that

$$
\phi(\mathcal{K})=n_{+}(\mathcal{K})-n_{-}(\mathcal{K}) .
$$

The problem is that the cotorsion is not invariant under deformations of the knot. In order to preserve topological invariance of the correlation function, one has to choose another definition of the composite operator $\left(\oint_{\mathcal{K}} A\right)^{2}$ by means of a framing. A framing of the knot consists of choosing another knot $\mathcal{K}^{f}$ around $\mathcal{K}$, specified by a normal vector field $n$. The cotorsion $\phi(\mathcal{K})$ then becomes

$$
\phi_{f}(\mathcal{K})=\frac{1}{4 \pi} \oint_{\mathcal{K}} \mathrm{d} x^{\mu} \oint_{\mathcal{K}^{f}} \mathrm{~d} y^{\nu} \epsilon_{\mu \nu \rho} \frac{(x-y)^{\rho}}{|x-y|^{3}}=\operatorname{lk}\left(\mathcal{K}, \mathcal{K}^{f}\right) .
$$

The correlation function that we obtain in this way is a topological invariant (since it only involves linking numbers) but the price that we have to pay is that our regularization depends on a set of integers $p_{\alpha}=\operatorname{lk}\left(\mathcal{K}_{\alpha}, \mathcal{K}_{\alpha}^{f}\right)$ (one for each knot). The correlation function (8) can now be computed, after choosing the framings, as follows:

$$
\left\langle\prod_{\alpha} \exp \left(n_{\alpha} \oint_{\mathcal{K}_{\alpha}} A\right)\right\rangle=\exp \left\{\frac{\pi \mathrm{i}}{k}\left(\sum_{\alpha} n_{\alpha}^{2} p_{\alpha}+\sum_{\alpha \neq \beta} n_{\alpha} n_{\beta} \operatorname{lk}\left(\mathcal{K}_{\alpha}, \mathcal{K}_{\beta}\right)\right)\right\} .
$$

This regularization is simply the 'point-splitting' method familiar in the context of quantum field theory.

Let us now consider Chern-Simons theory with gauge group $U(N)$, and suppose that we are interested in the computation of (8), in the context of perturbation theory. It is easy to see that self-contractions of the holonomies lead to the same kind of ambiguities that we found in the Abelian case, i.e. a choice of framing has to be made for each knot $\mathcal{K}_{\alpha}$. The only difference from the Abelian case is that the self-contraction of $\mathcal{K}_{\alpha}$ gives a group factor $\operatorname{Tr}_{R_{\alpha}}\left(T_{a} T_{a}\right)$, where $T_{a}$ is a basis of the Lie algebra (see, for example, Guadagnini et al. 1990). The precise result can be better stated as the effect on the correlation function (8) under a change of framing, and it says that, under a change of framing of $\mathcal{K}_{\alpha}$ by $p_{\alpha}$ units, the vacuum expectation value of the product of Wilson loops changes as follows (Witten 1989):

$$
W_{R_{1} \cdots R_{L}} \rightarrow \exp \left[2 \pi \mathrm{i} \sum_{\alpha=1}^{L} p_{\alpha} h_{R_{\alpha}}\right] W_{R_{1} \cdots R_{L}} .
$$

In this equation, $h_{R}$ is the conformal weight of the WZW primary field corresponding to the representation $R$. One can write (41) as 


$$
h_{R}=\frac{C_{R}}{2(k+N)},
$$

where $C_{R}=\operatorname{Tr}_{R}\left(T_{a} T_{a}\right)$ is the quadratic Casimir in the representation $R$. For $U(N)$ one has

$$
C_{R}=N \ell(R)+\kappa_{R}
$$

where $\ell(R)$ is the total number of boxes in the tableau, and

$$
\kappa_{R}=\ell(R)+\sum_{i}\left(l_{i}^{2}-2 i l_{i}\right)
$$

In terms of the variables (12) the change under framing (71) can be written as

$$
W_{R_{1} \cdots R_{L}} \rightarrow q^{\frac{1}{2} \sum_{\alpha=1}^{L} \kappa_{R_{\alpha}} p_{\alpha}} \lambda^{\frac{1}{2} \sum_{\alpha=1}^{L} \ell\left(R_{\alpha}\right) p_{\alpha}} W_{R_{1} \cdots R_{L}}
$$

Therefore, the evaluation of vacuum expectation values of Wilson loop operators in Chern-Simons theory depends on a choice of framing for knots. It turns out that for knots and links in $\mathbf{S}^{3}$, there is a standard or canonical framing, defined by requiring that the self-linking number is zero. The expressions we have given before for the Chern-Simons invariant of the unknot and the Hopf link are all in the standard framing. Once the value of the invariant is known in the standard framing, the value in any other framing specified by non-zero integers $p_{\alpha}$ can be easily obtained from (71).

\subsection{The 1/N Expansion in Chern-Simons Theory}

As we explained above, the perturbative series of Chern-Simons theory around the trivial connection can be re-expressed in terms of fatgraphs. In particular, one should be able to study the free energy of Chern-Simons theory on the three-sphere in the $1 / N$ expansion, i.e. to expand it as in (30) and to resum all fatgraphs of fixed genus in this expansion to obtain the quantities $F_{g}(t)$. In this section we will obtain closed expressions for $F_{g, h}$ and $F_{g}(t)$ in the case of Chern-Simons theory defined on $\mathbf{S}^{3}$, following Gopakumar and Vafa (1998a, 1999). For earlier work on the $1 / N$ expansion of Chern-Simons theory, see Camperi et al. (1990), Periwal (1993) and Correale and Guadagnini (1994).

A direct computation of $F_{g, h}$ from perturbation theory is difficult, since it involves the evaluation of integrals of products of propagators over the threesphere. However, in the case of $\mathbf{S}^{3}$ we have an exact expression for the partition function and we can expand it in both $x$ and $N$ to obtain the coefficients of (30). The partition function of CS with gauge group $U(N)$ on the three-sphere can be obtained from the formula (46) for $S U(N)$ after multiplying it by an overall $N^{1 / 2} /(k+N)^{1 / 2}$, which is the partition function of the $U(1)$ factor. The final result is 


$$
Z=\frac{1}{(k+N)^{N / 2}} \prod_{\alpha>0} 2 \sin \left(\frac{\pi(\alpha \cdot \rho)}{k+N}\right) .
$$

Using the explicit description of the positive roots of $S U(N)$, one gets

$$
F=\log Z=-\frac{N}{2} \log (k+N)+\sum_{j=1}^{N-1}(N-j) \log \left[2 \sin \frac{\pi j}{k+N}\right] .
$$

We can now write the sin as

$$
\sin \pi z=\pi z \prod_{n=1}^{\infty}\left(1-\frac{z^{2}}{n^{2}}\right),
$$

and we find that the free energy is the sum of two parts. We will call the first one the non-perturbative part:

$$
F^{\mathrm{np}}=-\frac{N^{2}}{2} \log (k+N)+\frac{1}{2} N(N-1) \log 2 \pi+\sum_{j=1}^{N-1}(N-j) \log j,
$$

and the other part will be called the perturbative part:

$$
F^{\mathrm{p}}=\sum_{j=1}^{N}(N-j) \sum_{n=1}^{\infty} \log \left[1-\frac{j^{2} g_{s}^{2}}{4 \pi^{2} n^{2}}\right]
$$

where we have denoted

$$
g_{s}=\frac{2 \pi}{k+N},
$$

which, as we will see later, coincides with the open string coupling constant under the gauge/string theory duality.

To see that (79) has a non-perturbative origin, we rewrite it as

$$
F^{\mathrm{np}}=\log \frac{\left(2 \pi g_{s}\right)^{\frac{1}{2} N^{2}}}{\operatorname{vol}(U(N))},
$$

where we used the explicit formula

$$
\operatorname{vol}(U(N))=\frac{(2 \pi)^{\frac{1}{2} N(N+1)}}{G_{2}(N+1)},
$$

and $G_{2}(N)$ is Barnes function. This indeed corresponds to the volume of the gauge group in the one-loop contribution (17), where $A^{(c)}$ is in this case the trivial flat connection. Therefore, $F^{\mathrm{np}}$ is the $\log$ of the prefactor of the path integral, which is not captured by Feynman diagrams. 
Let us now work out the perturbative part (80), following Gopakumar and Vafa (1998a, 1999). By expanding the log, using that $\sum_{n=1}^{\infty} n^{-2 k}=\zeta(2 k)$, and the formula

$$
\sum_{j=1}^{N} j^{k}=\frac{1}{k+1} \sum_{l=1}^{k+1}(-1)^{k-l+1}\left(\begin{array}{c}
k+1 \\
l
\end{array}\right) B_{k+1-l} N^{l}
$$

where $B_{n}$ are Bernoulli numbers, we find that (80) can be written as

$$
F^{\mathrm{p}}=\sum_{g=0}^{\infty} \sum_{h=2}^{\infty} F_{g, h}^{\mathrm{p}} g_{s}^{2 g-2+h} N^{h}
$$

where $F_{g, h}^{\mathrm{p}}$ is given by:

$$
\begin{aligned}
& F_{0, h}^{\mathrm{p}}=-\frac{\left|B_{h-2}\right|}{(h-2) h !}, \quad h \geq 4, \\
& F_{1, h}^{\mathrm{p}}=\frac{1}{12} \frac{\left|B_{h}\right|}{h h !} .
\end{aligned}
$$

Notice that $F_{0, h}^{\mathrm{p}}$ vanishes for $h \leq 3$. For $g \geq 2$ one obtains

$$
F_{g, h}^{\mathrm{p}}=\frac{\zeta(2 g-2+h)}{(2 \pi)^{2 g-3+h}}\left(\begin{array}{c}
2 g-3+h \\
h
\end{array}\right) \frac{B_{2 g}}{2 g(2 g-2)} .
$$

This gives the contribution of connected diagrams with two loops and beyond to the free energy of Chern-Simons theory on the sphere. The nonperturbative part also admits an asymptotic expansion that can be easily worked out by expanding the Barnes function that appears in the volume factor (Periwal 1993; Ooguri and Vafa 2002). One gets:

(88) $F^{\mathrm{np}}=\frac{N^{2}}{2}\left(\log \left(N g_{s}\right)-\frac{3}{2}\right)-\frac{1}{12} \log N+\zeta^{\prime}(-1)+\sum_{g=2}^{\infty} \frac{B_{2 g}}{2 g(2 g-2)} N^{2-2 g}$.

In order to find $F_{g}(t)$ we have to sum over the holes, as in (33). The 't Hooft parameter is given by $t=x N=\mathrm{i} g_{s} N$, and

$$
F_{g}^{\mathrm{p}}(t)=\sum_{h=1}^{\infty} F_{g, h}^{\mathrm{p}}(-\mathrm{i} t)^{h}
$$

Let us first focus on $g \geq 2$. To perform the sum explicitly, we again write the $\zeta$ function as $\zeta(2 g-2+2 p)=\sum_{n=1}^{\infty} n^{2-2 g-2 p}$, and use the binomial series,

$$
\frac{1}{(1-z)^{q}}=\sum_{n=0}^{\infty}\left(\begin{array}{c}
q+n-1 \\
n
\end{array}\right) z^{n}
$$


to obtain:

$$
F_{g}^{\mathrm{p}}(t)=\frac{(-1)^{g}\left|B_{2 g} B_{2 g-2}\right|}{2 g(2 g-2)(2 g-2) !}+\frac{B_{2 g}}{2 g(2 g-2)} \sum_{n \in \mathbf{Z}}{ }^{\prime} \frac{1}{(-\mathrm{i} t+2 \pi n)^{2 g-2}},
$$

where ' means that we omit $n=0$. Now we notice that, if we write

$$
F^{\mathrm{np}}=\sum_{g=0}^{\infty} F_{g}^{\mathrm{np}}(t) g_{s}^{2 g-2},
$$

then for, $g \geq 2, F_{g}^{\mathrm{np}}(t)=B_{2 g} /\left(2 g(2 g-2)(-\mathrm{i} t)^{2 g-2}\right.$, which is precisely the $n=0$ term missing in (91). We then define:

$$
F_{g}(t)=F_{g}^{\mathrm{p}}(t)+F_{g}^{\mathrm{np}}(t) .
$$

Finally, since

$$
\sum_{n \in \mathbf{Z}} \frac{1}{n+z}=\frac{2 \pi \mathrm{i}}{1-e^{-2 \pi \mathrm{i} z}}
$$

by taking derivatives w.r.t. $z$ we can write

$$
F_{g}(t)=\frac{(-1)^{g}\left|B_{2 g} B_{2 g-2}\right|}{2 g(2 g-2)(2 g-2) !}+\frac{\left|B_{2 g}\right|}{2 g(2 g-2) !} \operatorname{Li}_{3-2 g}\left(e^{-t}\right),
$$

again for $g \geq 2$. The function $\mathrm{Li}_{j}$ appearing in this equation is the polylogarithm of index $j$, defined by

$$
\operatorname{Li}_{j}(x)=\sum_{n=1}^{\infty} \frac{x^{n}}{n^{j}}
$$

The computation for $g=0,1$ is very similar, and one obtains:

$$
\begin{aligned}
& F_{0}(t)=-\frac{t^{3}}{12}+\frac{\pi^{2} t}{6}+\zeta(3)+\operatorname{Li}_{3}\left(e^{-t}\right), \\
& F_{1}(t)=\frac{t}{24}+\frac{1}{12} \log \left(1-e^{-t}\right) .
\end{aligned}
$$

This gives the resummed functions $F_{g}(t)$ introduced in (33) for all $g \geq 0$.

\section{Topological Strings}

In this section we give a rough presentation of Gromov-Witten invariants. Detailed definitions and constructions can be found for example in Cox and Katz (1999). 


\subsection{Topological Strings and Gromov-Witten Invariants}

In order to define Gromov-Witten invariants, the starting point is the moduli space of possible metrics (or equivalently, complex structures) on a Riemann surface with punctures, which is the famous Deligne-Mumford space $\bar{M}_{g, n}$ of $n$-pointed stable curves (the definition of what stable means can be found for example in Harris and Morrison 1998). Let $X$ be a Kähler manifold. The relevant moduli space in Gromov-Witten theory is denoted by

$$
\bar{M}_{g, n}(X, \beta)
$$

where $\beta \in H_{2}(X)$. This is a generalization of $\bar{M}_{g, n}$, and depends on a choice of a two-homology class $\beta$ in $X$. Very roughly, a point in $\bar{M}_{g, n}(X, \beta)$ can be written as $\left(f, \Sigma_{g}, p_{1}, \cdots, p_{n}\right)$ and is given by (a) a point in $\bar{M}_{g, n}$, i.e. a Riemann surface with $n$ punctures, $\left(\Sigma_{g}, p_{1}, \cdots, p_{n}\right)$, together with a choice of complex structure on $\Sigma_{g}$, and (b) a map $f: \Sigma_{g} \rightarrow X$ that is holomorphic with respect to this choice of complex structure and such that $f_{*}\left[\Sigma_{g}\right]=\beta$. The set of all such points forms a good moduli space provided a certain number of conditions are satisfied (see for example Cox and Katz (1999) and Hori et al. (2003) for a detailed discussion of these issues). $\bar{M}_{g, n}(X, \beta)$ is the basic moduli space we will need in the theory of topological strings. Its complex virtual dimension is given by

$$
(1-g)(d-3)+n+\int_{\Sigma_{g}} f^{*}\left(c_{1}(X)\right) .
$$

We also have two natural maps

$$
\begin{aligned}
& \pi_{1}: \bar{M}_{g, n}(X, \beta) \longrightarrow X^{n}, \\
& \pi_{2}: \bar{M}_{g, n}(X, \beta) \longrightarrow \bar{M}_{g, n} .
\end{aligned}
$$

The first map is easy to define: given a point $\left(f, \Sigma_{g}, p_{1}, \cdots, p_{n}\right)$ in $\bar{M}_{g, n}(X, \beta)$, we just compute $\left(f\left(p_{1}\right), \cdots, f\left(p_{n}\right)\right)$. The second map essentially sends $\left(f, \Sigma_{g}\right.$, $\left.p_{1}, \cdots, p_{n}\right)$ to $\left(\Sigma_{g}, p_{1}, \cdots, p_{n}\right)$, i.e. forgets the information about the map and keeps the information about the punctured curve.

We can now formally define the Gromov-Witten invariant $I_{g, n, \beta}$ as follows. Let us consider cohomology classes $\phi_{1}, \cdots, \phi_{n}$ in $H^{*}(X)$. If we pull back their tensor product to $H^{*}\left(\bar{M}_{g, n}(X, \beta)\right)$ via $\pi_{1}$, we get a differential form on the moduli space of maps that we can integrate (as long as there is a well-defined fundamental class for this space):

$$
I_{g, n, \beta}\left(\phi_{1}, \cdots, \phi_{n}\right)=\int_{\bar{M}_{g, n}(X, \beta)} \pi_{1}^{*}\left(\phi_{1} \otimes \cdots \otimes \phi_{n}\right) .
$$

The Gromov-Witten invariant $I_{g, n, \beta}\left(\phi_{1}, \cdots, \phi_{n}\right)$ vanishes unless the degree of the form equals the dimension of the moduli space. Therefore, we have the following constraint: 


$$
\frac{1}{2} \sum_{i=1}^{n} \operatorname{deg}\left(\phi_{i}\right)=(1-g)(d-3)+n+\int_{\Sigma_{g}} f^{*}\left(c_{1}(X)\right) .
$$

Notice that Calabi-Yau threefolds play a special role in the theory, since for those targets the virtual dimension only depends on the number of punctures, and therefore the above condition is always satisfied if the forms $\phi_{i}$ have degree 2 .

When $n=0$, one gets an invariant $I_{g, 0, \beta}$ that does not require any insertions. This is the Gromov-Witten invariant on which we will focus, and we will denote it by $N_{g, \beta}$. Notice that these invariants are in general rational, due to the orbifold character of the moduli spaces involved. It is very convenient to introduce the generating functional of these invariants at fixed genus. This is defined as follows. First, choose a basis $\left[\Sigma_{i}\right] \in H_{2}(X)$ in such a way that

$$
\beta=\sum_{i=1}^{h^{1,1}(X)} \beta_{i}\left[\Sigma_{i}\right] .
$$

We also introduce $h^{1,1}(X)$ complexified Kähler parameters $t_{i}$. They are defined as

$$
t_{i}=\int_{\Sigma_{i}} \omega
$$

In this equation, $\omega$ is the complexified Kähler class,

$$
\omega=J+\mathrm{i} B,
$$

where $J$ is the Kähler class and $B$ is the B-field. Finally, we introduce

$$
\beta \cdot t=\sum_{i=1}^{h^{1,1}(X)} \beta_{i} t_{i}=\int_{\beta} \omega .
$$

With these ingredients, we define the topological string amplitude at genus $g$ as the generating functional

$$
F_{g}(t)=\sum_{\beta \in H_{2}(X)} N_{g, \beta} \mathrm{e}^{-\beta \cdot t} .
$$

The total topological string amplitude sums this to all genera,

$$
F\left(g_{s}, t\right)=\sum_{g=0}^{\infty} F_{g}(t) g_{s}^{2 g-2} .
$$

It is also convenient to consider the exponentiated functional, which is called the topological string partition function,

$$
Z\left(g_{s}, t\right)=\exp F\left(g_{s}, t\right) .
$$


An important goal in Gromov-Witten theory is to provide effective tools for the computation of these quantities. The main reason why physics is useful in doing this is because the $F_{g}(t)$ are couplings in type II string theory, and can be also obtained as free energies of topological string theory, a topological version of string theory which is obtained by coupling topological sigma models to topological gravity (hence the name of topological string quantities for these quantities). For an exposition of some of the relevant physics background, see Mariño (2005).

\subsection{Integrality Properties and Gopakumar-Vafa Invariants}

It was shown by Gopakumar and Vafa (1998b) that the total free energy $F\left(g_{s}, t\right)$ can be expressed in terms of integer numbers $n_{\beta}^{g}$ as follows

$$
F\left(g_{s}, t\right)=\sum_{g=0}^{\infty} \sum_{\beta} \sum_{d=1}^{\infty} n_{\beta}^{g} \frac{1}{d}\left(2 \sin \frac{d g_{s}}{2}\right)^{2 g-2} Q^{d \beta} .
$$

The integers $n_{\beta}^{g}$ are known as Gopakumar-Vafa invariants. They are true invariants of the Calabi-Yau manifold $X$, in the sense that they do not depend on smooth deformations of the target geometry, This is in contrast to the quantities $n_{\beta}^{\left(j_{L}, j_{R}\right)}$, which do depend on deformations. As usual, by tracing over a non-invariant quantity with signs we obtain an invariant quantity.

The structure result (110) implies that Gromov-Witten invariants of closed strings, which are in general rational, can be written in terms of these integer invariants. In fact, by knowing the Gromov-Witten invariants $N_{g, \beta}$ we can explicitly compute the Gopakumar-Vafa invariants from (110) (an explicit inversion formula can be found in Bryan and Pandharipande 2001). By expanding in $g_{s}$, it is easy to show that the Gopakumar-Vafa formula (110) predicts the following expression for $F_{g}(t)$ :

$$
F_{g}(t)=\sum_{\beta}\left(\frac{\left|B_{2 g}\right| n_{\beta}^{0}}{2 g(2 g-2) !}+\frac{2(-1)^{g} n_{\beta}^{2}}{(2 g-2) !} \pm \cdots-\frac{g-2}{12} n_{\beta}^{g-1}+n_{\beta}^{g}\right) \operatorname{Li}_{3-2 g}\left(Q^{\beta}\right),
$$

where $\mathrm{Li}_{j}$ is the polylogarithm defined in (96). The appearance of the polylogarithm of order $3-2 g$ in $F_{g}$ was first predicted from type IIA/heterotic string duality by Mariño and Moore (1999).

The structure found by Gopakumar and Vafa solves some longstanding issues in the theory of Gromov-Witten invariants, in particular the enumerative meaning of the invariants. Two obstructions to finding obvious enumerative meaning to Gromov-Witten invariants are multicovering and bubbling. Multicovering arises as follows. Suppose one finds a holomorphic map $x: \mathbb{P}^{1} \rightarrow X$ in genus zero and in the class $\beta$. Then, simply by composing this with a degree $d$ cover $\mathbb{P}^{1} \rightarrow \mathbb{P}^{1}$, one can find another holomorphic map in the class $d \beta$. Therefore, at every degree, in order to count the actual number of 'primitive' 
holomorphic curves, one should subtract from the corresponding GromovWitten invariant the contributions coming from multicovering of curves with lower degree. Another geometric effect that has to be taken into account is bubbling (see, for example, Bershadsky et al. 1993, 1994). Imagine that one finds a map $x: \Sigma_{g} \rightarrow X$ from a genus $g$ Riemann surface to a Calabi-Yau threefold. By gluing to $\Sigma_{g}$ a small Riemann surface of genus $h$, and making it very small, one can find an approximate holomorphic map from a Riemann surface whose genus is topologically $g+h$. This means that 'primitive' maps at genus $g$ contribute to all genera $g^{\prime}>g$, and in order to count curves properly one should take this effect into account.

The formula (111) gives a precise answer to these questions. Consider, for example, the structure of $F_{0}$. According to the above formula, the contribution of a Gopakumar-Vafa invariant is given by the function $\mathrm{Li}_{3}$ :

$$
\sum_{d=1}^{\infty} \frac{Q^{d \beta}}{d^{3}} .
$$

This gives the contribution of all the multicoverings of a given 'primitive' curve, where $d$ is the degree of the multicovering. In addition, it says that each cover has a weight $1 / d^{3}$. Therefore, the invariant $n_{\beta}^{0}$ corresponds to primitive holomorphic maps, and the non-integrality of genus-zero GromovWitten invariants is due to the effects of multicovering. The multicovering phenomenon in genus 0 was found experimentally in Candelas et al. (1991) and later derived in the context of Gromov-Witten theory by Aspinwall and Morrison (1993). The structure result of Gopakumar and Vafa also predicts that the multicovering of degree $d$ of a genus $g$ curve contributes with a weight $d^{3-2 g}$ (coming from $\mathrm{Li}_{3-2 g}$ ). Moreover, the formula (111) implies that a genus $h<g$ Gopakumar-Vafa invariant contributes to $F_{g}(t)$ with a precise weight, and this corresponds to the bubbling effects we mentioned before. For example, a genus 0 Gopakumar-Vafa invariant contributes to $F_{g}$ with a weight $\left|B_{2 g}\right| /(2 g(2 g-2) !)$.

\subsection{Open Topological Strings}

So far we have discussed the Gromov-Witten theory for the case of closed Riemann surfaces, but the theory can be (at least formally) extended to the open case. The natural starting point is to consider maps from a Riemann surface $\Sigma_{g, h}$ of genus $g$ with $h$ holes. Such models were analysed in detail by Witten (1995). The main issue is, of course, to specify boundary conditions for the maps $f: \Sigma_{g, h} \rightarrow X$. It turns out that the relevant boundary conditions are Dirichlet and given by Lagrangian submanifolds of the Calabi-Yau $X$. A Lagrangian submanifold $\mathcal{L}$ is a cycle on which the Kähler form vanishes:

$$
\left.J\right|_{\mathcal{L}}=0 .
$$


If we denote by $C_{i}, i=1, \cdots, h$, the boundaries of $\Sigma_{g, h}$ we have to pick a Lagrangian submanifold $\mathcal{L}$, and consider holomorphic maps such that

$$
f\left(C_{i}\right) \subset \mathcal{L} .
$$

Once boundary conditions have been specified, we look at holomorphic maps from open Riemann surfaces of genus $g$ and with $h$ holes to the CalabiYau $X$, with Dirichlet boundary conditions specified by $\mathcal{L}$. These holomorphic maps are called open string instantons, and can also be classified topologically. The topological sector of an open string instanton is given by two different kinds of data: the boundary part and the bulk part. For the bulk part, the topological sector is labelled by relative homology classes, since we are requiring the boundaries of $f_{*}\left[\Sigma_{g, h}\right]$ to end on $\mathcal{L}$. Therefore, we will set

$$
f_{*}\left[\Sigma_{g, h}\right]=\beta \in H_{2}(X, \mathcal{L}) .
$$

To specify the topological sector of the boundary, we will assume that $b_{1}(\mathcal{L})=1$, so that $H_{1}(\mathcal{L})$ is generated by a non-trivial one-cycle $\gamma$. We then have

$$
f_{*}\left[C_{i}\right]=w_{i} \gamma, \quad w_{i} \in \mathbf{Z}, \quad i=1, \cdots, h,
$$

in other words, $w_{i}$ is the winding number associated to the map $f$ restricted to $C_{i}$. We will collect these integers into a single $h$-uple denoted by $w=$ $\left(w_{1}, \cdots, w_{h}\right)$.

The free energy of open topological string theory at fixed genus and boundary data $w$, which we denote by $F_{w, g}(t)$, can be computed as a sum over open string instantons labelled by the bulk classes:

$$
F_{w, g}(t)=\sum_{\beta} F_{w, g, \beta} \mathrm{e}^{-\beta \cdot t} .
$$

In this equation, the sum is over relative homology classes $\beta \in H_{2}(X, \mathcal{L})$. The quantities $F_{w, g, \beta}$ are open Gromov-Witten invariants. They 'count' in an appropriate sense the number of holomorphically embedded Riemann surfaces of genus $g$ in $X$ with Lagrangian boundary conditions specified by $\mathcal{L}$, and in the class represented by $\beta, w$. They are in general rational numbers. In contrast to conventional Gromov-Witten invariants, a rigorous theory of open GromovWitten invariants is not yet available. However, localization techniques make it possible to compute them in some situations (Katz and Liu 2002; Li and Song 2002; Graber and Zaslow 2002; Mayr 2002).

In order to consider all topological sectors, we have to introduce the string coupling constant $g_{s}$, which takes care of the genus, as well as a Hermitian $M \times M$ matrix $V$, which takes care of the different winding numbers $w$. The total free energy is defined by

$$
F(V)=\sum_{g=0}^{\infty} \sum_{h=1}^{\infty} \sum_{w_{1}, \cdots, w_{h}} \frac{\mathrm{i}^{h}}{h !} g_{s}^{2 g-2+h} F_{w, g}(t) \operatorname{Tr} V^{w_{1}} \cdots \operatorname{Tr} V^{w_{h}} .
$$


The factor $\mathrm{i}^{h}$ is introduced for convenience, while $h$ ! is a symmetry factor which takes into account that the holes are indistinguishable. Notice that, in order to distinguish all possible topological sectors, one has to take $V$ to have infinite rank, and formally we can think about the different traces in (118) as symmetric functions in an infinite number of variables.

If the winding numbers $w_{i}$ in (118) are all positive, the product of traces of $V$ in (118) can be written in terms of $\operatorname{Tr}_{R} V$ for representations $R$ with a small number of boxes:

$$
F(V)=\sum_{R} F_{R}\left(g_{s}, t\right) \operatorname{Tr}_{R} V,
$$

Negative winding numbers can be introduced through another set of representations. We have also assumed that the boundary conditions are specified by a single Lagrangian submanifold with a single non-trivial one-cycle. When there are more one-cycles in the geometry, say $L$, providing possible boundary conditions for the open strings, the above formalism has to be generalized in an obvious way: one needs to specify $L$ sets of winding numbers $w^{(\alpha)}$, and the generating functional (119) depends on $L$ different matrices $V_{\alpha}, \alpha=1, \cdots, L$. The total partition function is the formal exponential of the total free energy and it has the structure

$$
Z\left(V_{i}\right)=\sum_{R_{1}, \cdots, R_{2 L}} Z_{R_{1} \cdots R_{2 L}}\left(g_{s}, t\right) \prod_{\alpha=1}^{2 L} \operatorname{Tr}_{R_{\alpha}} V_{\alpha},
$$

where the $R_{2 \alpha-1}, R_{2 \alpha}$ correspond to positive and negative winding numbers, respectively, for the $\alpha$-th cycle.

\section{Toric Geometry and Calabi-Yau Threefolds}

\subsection{Non-Compact Calabi-Yau Geometries: An Introduction}

One of the main insights in the study of Gromov-Witten theory on Calabi-Yau threefolds is that the simplest models to study are associated to non-compact Calabi-Yau geometries based on manifolds of lower dimension. To construct these geometries, we start with complex manifolds in one or two complex dimensions, which in general will have a non-zero first Chern class. We then consider vector bundles over them (with the appropriate rank and curvature) that lead to a total three-dimensional space with zero first Chern class. In this way, we obtain Calabi-Yau threefolds whose non-trivial geometry is encoded in a lower-dimensional manifold, and therefore they are easier to study.

Let us first consider non-compact Calabi-Yau manifolds whose building block is a one-dimensional compact manifold. These manifolds will be given by a Riemann surface together with an appropriate bundle over it, and geometrically they can be regarded as the local geometry of an embedded Riemann 
surface in a general Calabi-Yau space. Indeed, consider a Riemann surface $\Sigma_{g}$ holomorphically embedded inside a Calabi-Yau threefold $X$, and let us look at the holomorphic tangent bundle of $X$ restricted to $\Sigma_{g}$. We have

$$
\left.T X\right|_{\Sigma_{g}}=T \Sigma_{g} \oplus \mathcal{N}_{\Sigma_{g}}
$$

where $\mathcal{N}_{\Sigma_{g}}$ is a holomorphic rank-two complex vector bundle over $\Sigma_{g}$, called the normal bundle of $\Sigma_{g}$, and the Calabi-Yau condition $c_{1}(X)=0$ gives

$$
c_{1}\left(\mathcal{N}_{\Sigma_{g}}\right)=2 g-2 .
$$

The Calabi-Yau $X$ 'near $\Sigma_{g}$ ' then looks like the total space of the bundle

$$
\mathcal{N} \rightarrow \Sigma_{g},
$$

where $\mathcal{N}$ is regarded here as a rank-two bundle over $\Sigma_{g}$ satisfying (122). The non-compact space (123) is an example of a local Calabi-Yau threefold.

When $g=0$ and $\Sigma_{g}=\mathbb{P}^{1}$ it is possible to be more precise about the bundle $\mathcal{N}$. A theorem due to Grothendieck says that any holomorphic bundle over $\mathbb{P}^{1}$ splits into a direct sum of line bundles (for a proof, see for example Griffiths and Harris 1977, pp. 516-7). Line bundles over $\mathbb{P}^{1}$ are all of the form $\mathcal{O}(n)$, where $n \in \mathbf{Z}$. The bundle $\mathcal{O}(n)$ can be easily described in terms of two charts on $\mathbb{P}^{1}$ : the north-pole chart, with co-ordinates $z, \Phi$ for the base and the fibre, respectively, and the south-pole chart, with co-ordinates $z^{\prime}, \Phi^{\prime}$. The change of co-ordinates is given by

$$
z^{\prime}=1 / z, \quad \Phi^{\prime}=z^{-n} \Phi .
$$

We also have that $c_{1}(\mathcal{O}(n))=n$. We then find that local Calabi-Yau manifolds that are made out of a two-sphere together with a bundle over it are all of the form

$$
\mathcal{O}(-a) \oplus \mathcal{O}(a-2) \rightarrow \mathbb{P}^{1},
$$

since the degrees of the bundles have to sum up to -2 due to (122). An important case occurs when $a=1$. The resulting non-compact manifold,

$$
\mathcal{O}(-1) \oplus \mathcal{O}(-1) \rightarrow \mathbb{P}^{1},
$$

is called the resolved conifold for reasons that will be explained later.

We can also consider non-compact Calabi-Yau threefolds based on compact complex surfaces. Consider a complex surface $S$ embedded in a CalabiYau manifold $X$. As before, we can split the tangent bundle as

$$
\left.T X\right|_{S}=T S \oplus \mathcal{N}_{S},
$$

where the normal bundle $\mathcal{N}_{S}$ is now of rank one. The Calabi-Yau condition leads to 


$$
c_{1}\left(\mathcal{N}_{S}\right)=c_{1}\left(K_{S}\right),
$$

where $K_{S}$ is the canonical line bundle over $S$, and we used that $c_{1}(T S)=$ $-c_{1}\left(K_{S}\right)$. Therefore, we have $\mathcal{N}_{S}=K_{S}$. The Calabi-Yau $X$ 'near $S$ ' looks like the total space of the bundle

$$
K_{S} \rightarrow S
$$

This construction gives a whole family of non-compact Calabi-Yau manifolds that are also referred to as local Calabi-Yau manifolds. A well-known example is $S=\mathbb{P}^{2}$, the two-dimensional projective space, which leads to the CalabiYau manifold

$$
\mathcal{O}(-3) \rightarrow \mathbb{P}^{2},
$$

also known as local $\mathbb{P}^{2}$. Another important example is $S=\mathbb{P}^{1} \times \mathbb{P}^{1}$, which leads to local $\mathbb{P}^{1} \times \mathbb{P}^{1}$.

\subsection{Constructing Toric Calabi-Yau Manifolds}

Many of the examples of non-compact Calabi-Yau threefolds considered above are toric, i.e. they have the structure of a torus fibration, and can be constructed in a systematic way by a 'cut and paste' procedure. In this section we will develop these techniques, following the approach of Aganagic et al. (2005).

\section{$\mathbf{C}^{3}$}

The elementary building block for the technique we want to develop is a very simple non-compact Calabi-Yau threefold, namely $\mathbf{C}^{3}$. We will now exhibit its structure as a $\mathbf{T}^{2} \times \mathbb{R}$ fibration over $\mathbb{R}^{3}$, and we will encode this information in a simple trivalent, planar graph.

Let $z_{i}$ be complex co-ordinates on $\mathbf{C}^{3}, i=1,2,3$. We introduce three functions or Hamiltonians

$$
\begin{aligned}
& r_{\alpha}(z)=\left|z_{1}\right|^{2}-\left|z_{3}\right|^{2}, \\
& r_{\beta}(z)=\left|z_{2}\right|^{2}-\left|z_{3}\right|^{2}, \\
& r_{\gamma}(z)=\operatorname{Im}\left(z_{1} z_{2} z_{3}\right) .
\end{aligned}
$$

These Hamiltonians generate three flows on $\mathbf{C}^{3}$ via the standard symplectic form $\omega=\mathrm{i} \sum_{j} d z_{j} \wedge d \bar{z}_{j}$ on $\mathbf{C}^{3}$ and the Poisson brackets

$$
\partial_{v} z_{i}=\left\{r_{v}, z_{i}\right\}_{\omega}, \quad v=\alpha, \beta, \gamma .
$$

This gives the fibration structure that we were looking for: the base of the fibration, $\mathbb{R}^{3}$, is parameterized by the Hamiltonians (131), while the fibre $\mathbf{T}^{2} \times$ 
$\mathbb{R}$ is parameterized by the flows associated to the Hamiltonians. In particular, the $\mathbf{T}^{2}$ fibre is generated by the circle actions

$$
\mathrm{e}^{\alpha r_{\alpha}+\beta r_{\beta}}:\left(z_{1}, z_{2}, z_{3}\right) \rightarrow\left(\mathrm{e}^{\mathrm{i} \alpha} z_{1}, \mathrm{e}^{\mathrm{i} \beta} z_{2}, \mathrm{e}^{-i(\alpha+\beta)} z_{3}\right),
$$

while $r_{\gamma}$ generates the real line $\mathbb{R}$. We will call the cycle generated by $r_{\alpha}$ the $(0,1)$ cycle, and the cycle generated by $r_{\beta}$ the $(1,0)$ cycle.

Notice that the $(0,1)$ cycle degenerates over the subspace of $\mathbf{C}^{3}$ described by $z_{1}=0=z_{3}$, which is the subspace of the base $\mathbb{R}^{3}$ given by $r_{\alpha}=r_{\gamma}=0$, $r_{\beta} \geq 0$. Similarly, over $z_{2}=0=z_{3}$ the $(1,0)$-cycle degenerates over the subspace $r_{\beta}=r_{\gamma}=0$ and $r_{\alpha} \geq 0$. Finally, the one-cycle parameterized by $\alpha+\beta$ degenerates over $z_{1}=0=z_{2}$, where $r_{\alpha}-r_{\beta}=0=r_{\gamma}$ and $r_{\alpha} \leq 0$.

We will represent the $\mathbf{C}^{3}$ geometry by a graph that encodes the degeneration loci in the $\mathbb{R}^{3}$ base. In fact, it is useful to have a planar graph by taking $r_{\gamma}=0$ and drawing the lines in the $r_{\alpha}-r_{\beta}$ plane. The degeneration locus will then be straight lines described by the equation $p r_{\alpha}+q r_{\beta}=$ const. Over this line the $(-q, p)$ cycle of the $\mathbf{T}^{2}$ degenerates. Therefore we correlate the degenerating cycles unambiguously with the lines in the graph (up to $(q, p) \rightarrow(-q,-p))$. This yields the graph in Fig. 12, drawn in the $r_{\gamma}=0$ plane.

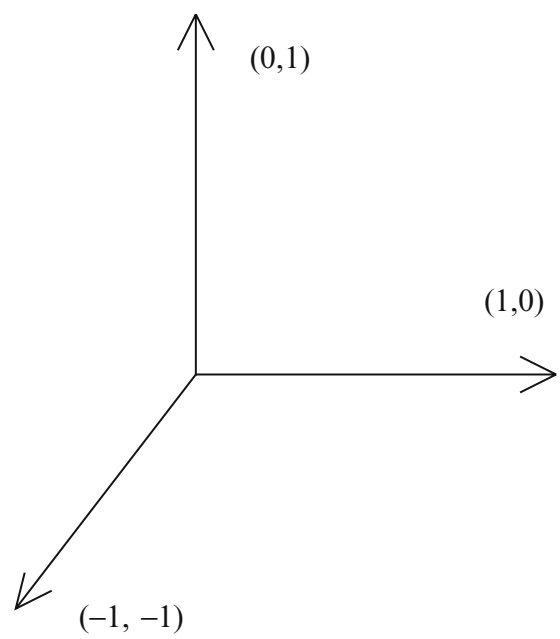

Fig. 12. This graph represents the degeneration locus of the $\mathbf{T}^{2} \times \mathbb{R}$ fibration of $\mathbf{C}^{3}$ in the base $\mathbb{R}^{3}$ parameterized by $\left(r_{\alpha}, r_{\beta}, r_{\gamma}\right)$

There is a symmetry in the $\mathbf{C}^{3}$ geometry that makes it possible to find other representations by different toric graphs. These graphs are characterized by three vectors $v_{i}$ that are obtained from those in Fig. 12 by an $\operatorname{SL}(2, \mathbf{Z})$ transformation. The vectors have to satisfy 


$$
\sum_{i} v_{i}=0
$$

The SL(2, Z ) symmetry is inherited from the $\mathrm{SL}(2, \mathbf{Z})$ symmetry of $\mathbf{T}^{2}$ that appeared in Sect. 2 in the context of Chern-Simons theory. In the above discussion the generators $H_{1}\left(\mathbf{T}^{2}\right)$ have been chosen to be the one-cycles associated to $r_{\alpha}$ and $r_{\beta}$, but there are other choices that differ from this one by an $\mathrm{SL}(2, \mathbf{Z})$ transformation on the $\mathbf{T}^{2}$. For example, we can choose $r_{\alpha}$ to generate a $(p, q)$ one-cycle and $r_{\beta}$ a $(t, s)$ one-cycle, provided that $p s-q t=1$. These different choices give different trivalent graphs. As we will see in the examples below, the construction of general toric geometries requires these more general graphs representing $\mathbf{C}^{3}$.

\section{The General Case}

The non-compact, toric Calabi-Yau threefolds that we will study can be described as symplectic quotients. Let us consider the complex linear space $\mathbf{C}^{N+3}$, described by $N+3$ co-ordinates $z_{1}, \cdots, z_{N+3}$, and let us introduce $N$ real equations of the form

$$
\mu_{A}=\sum_{j=1}^{N+3} Q_{A}^{j}\left|z_{j}\right|^{2}=t_{A}, \quad A=1, \cdots, N
$$

In this equation, $Q_{A}^{j}$ are integers satisfying

$$
\sum_{j=1}^{N+3} Q_{A}^{j}=0
$$

This condition is equivalent to $c_{1}(X)=0$, i.e. to the Calabi-Yau condition. We consider the action of the group $G_{N}=U(1)^{N}$ on the $z$ s where the $A$-th $U(1)$ acts on $z_{j}$ by

$$
z_{j} \rightarrow \exp \left(\mathrm{i} Q_{A}^{j} \alpha_{A}\right) z_{j}
$$

The space defined by (135), quotiented by the group action $G_{N}$,

$$
X=\bigcap_{A=1}^{N} \mu_{A}^{-1}\left(t_{A}\right) / G_{N}
$$

turns out to be a Calabi-Yau manifold (it can be seen that the condition (136) is equivalent to the Calabi-Yau condition). The $N$ parameters $t_{A}$ are Kähler moduli of the Calabi-Yau. This mathematical description of $X$ appears in the study of the two-dimensional linear sigma model with $\mathcal{N}=(2,2)$ supersymmetry (Witten 1993). The theory has $N+3$ chiral fields, whose lowest components are the $z \mathrm{~s}$ and are charged under $N$ vector multiplets with 
charges $Q_{A}^{j}$. The equations (135) are the D-term equations, and after dividing by the $U(1)^{N}$ gauge group we obtain the Higgs branch of the theory.

The Calabi-Yau manifold $X$ defined in (137) can be described by $\mathbf{C}^{3}$ geometries glued together in an appropriate way. Since each of these $\mathbf{C}^{3} \mathbf{s}$ is represented by the trivalent vertex depicted in Fig. 12, we will be able to encode the geometry of (137) into a trivalent graph. In order to provide this description, we must first find a decomposition of the set of all co-ordinates $\left\{z_{j}\right\}_{j=1}^{N+3}$ into triplets $U_{a}=\left(z_{i_{a}}, z_{j_{a}}, z_{k_{a}}\right)$ that correspond to the decomposition of $X$ into $\mathbf{C}^{3}$ patches. We pick one of the patches and we associate to it two Hamiltonians $r_{\alpha}, r_{\beta}$ as we did for $\mathbf{C}^{3}$ before. These two co-ordinates will be global co-ordinates in the base $\mathbb{R}^{3}$, therefore they will generate a globally defined $\mathbf{T}^{2}$ fibre. The third co-ordinate in the base is $r_{\gamma}=\operatorname{Im}\left(\prod_{j=1}^{N+3} z_{j}\right)$, which is manifestly gauge invariant and moreover, patch by patch, can be identified with the co-ordinate used in the $\mathbf{C}^{3}$ example above. Equation (135) can then be used to find the action of $r_{\alpha, \beta}$ on the other patches.

We will now exemplify this procedure with two important examples: the resolved conifold and the local $\mathbb{P}^{2}$ geometry, which were introduced before as local Calabi-Yau geometries.

Example. The resolved conifold. The resolved conifold (126) has a description of the form (137), with $N=1$. There is only one constraint given by

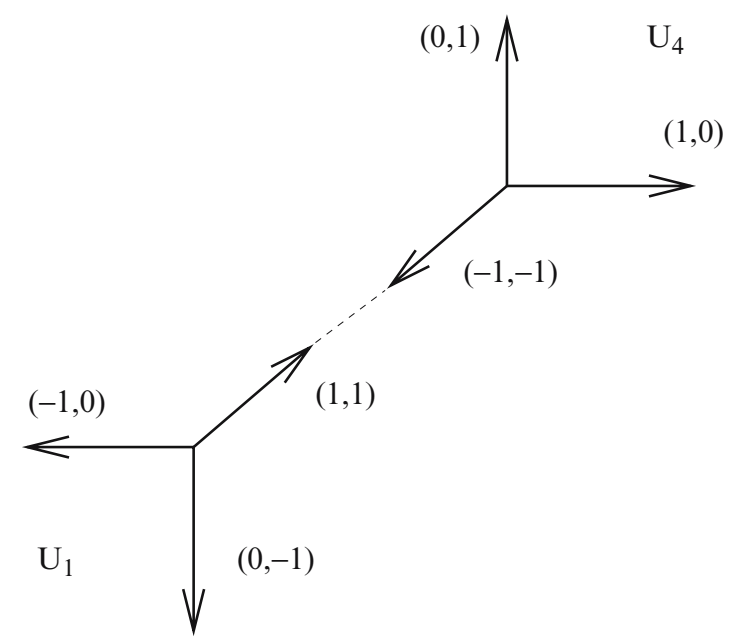

Fig. 13. The graph associated to the resolved conifold $\mathcal{O}(-1) \oplus \mathcal{O}(-1) \rightarrow \mathbb{P}^{1}$. This manifold is made out of two $\mathbf{C}^{3}$ patches glued through a common edge

$$
\left|z_{1}\right|^{2}+\left|z_{4}\right|^{2}-\left|z_{2}\right|^{2}-\left|z_{3}\right|^{2}=t
$$

and the $U(1)$ group acts as

$$
z_{1}, z_{2}, z_{3}, z_{4} \rightarrow \mathrm{e}^{\mathrm{i} \alpha} z_{1}, \mathrm{e}^{-\mathrm{i} \alpha} z_{2}, \mathrm{e}^{-\mathrm{i} \alpha} z_{3}, \mathrm{e}^{\mathrm{i} \alpha} z_{4}
$$


Notice that, for $z_{2}=z_{3}=0,(138)$ describes a $\mathbb{P}^{1}$ whose area is proportional to $t$. Therefore, $\left(z_{1}, z_{4}\right)$ can be taken as homogeneous co-ordinates of the $\mathbb{P}^{1}$ that is the basis of the fibration, while $z_{2}, z_{3}$ can be regarded as co-ordinates for the fibres.

Let us now give a description in terms of $\mathbf{C}^{3}$ patches glued together. The first patch will be defined by $z_{4} \neq 0$. Using (138) we can solve for the modulus of $z_{4}$ in terms of the other co-ordinates, and using the $U(1)$ action we can gauge away its phase. Therefore, the patch will be parameterized by $U_{4}=\left(z_{1}, z_{2}, z_{3}\right)$. The Hamiltonians will be, in this case,

$$
\begin{aligned}
& r_{\alpha}(z)=\left|z_{2}\right|^{2}-\left|z_{1}\right|^{2}, \\
& r_{\beta}(z)=\left|z_{3}\right|^{2}-\left|z_{1}\right|^{2},
\end{aligned}
$$

which generate the actions

$$
\mathrm{e}^{\alpha r_{\alpha}+\beta r_{\beta}}:\left(z_{1}, z_{2}, z_{3}\right) \rightarrow\left(\mathrm{e}^{-\mathrm{i}(\alpha+\beta)} z_{1}, \mathrm{e}^{\mathrm{i} \alpha} z_{2}, \mathrm{e}^{\mathrm{i} \beta} z_{3}\right) .
$$

This patch will be represented by the same graph that we found for $\mathbf{C}^{3}$. The other patch will be defined by $z_{1} \neq 0$, therefore we can write it as $U_{1}=$ $\left(z_{4}, z_{2}, z_{3}\right)$. However, in this patch $z_{1}$ is no longer a natural co-ordinate, but we can use (138) to rewrite the Hamiltonians as

$$
\begin{aligned}
& r_{\alpha}(z)=\left|z_{4}\right|^{2}-\left|z_{3}\right|^{2}-t, \\
& r_{\beta}(z)=\left|z_{4}\right|^{2}-\left|z_{2}\right|^{2}-t,
\end{aligned}
$$

generating the action

$$
\mathrm{e}^{\alpha r_{\alpha}+\beta r_{\beta}}:\left(z_{4}, z_{2}, z_{3}\right) \rightarrow\left(\mathrm{e}^{\mathrm{i}(\alpha+\beta)} z_{4}, \mathrm{e}^{-\mathrm{i} \beta} z_{2}, \mathrm{e}^{-\mathrm{i} \alpha} z_{3}\right) .
$$

The degeneration loci in this patch are the following: (1) $z_{4}=0=z_{2}$, corresponding to the line $r_{\beta}=-t$ where a $(-1,0)$ cycle degenerates; $(2)$ $z_{4}=0=z_{3}$, corresponding to the line $r_{\alpha}=-t$, and with a $(0,1)$ cycle degenerating; (3) finally, $z_{2}=0=z_{3}$, where $r_{\alpha}-r_{\beta}=0$, and a $(1,1)$ cycle degenerates. This patch is identical to the first one, and they are joined together through the common edge where $z_{2}=0=z_{3}$. The full construction is represented in Fig. 13. Notice that the common edge of the graphs represents the $\mathbb{P}^{1}$ of the resolved conifold: along this edge, one of the $\mathbf{S}^{1} \mathbf{S}$ of $\mathbf{T}^{2}$ has degenerated, while the other only degenerates at the endpoints. An $\mathbf{S}^{1}$ fibration of an interval that degenerates at its endpoints is simply a two-sphere. The length of the edge is $t$, the Kähler parameter associated to the $\mathbb{P}^{1}$.

Example. Local $\mathbb{P}^{2}$. Let us now consider a more complicated example, namely local $\mathbb{P}^{2}$, which is the total space of the bundle (130). We can describe it again as in (137) with $N=1$. There are four complex variables, $z_{0}, \cdots, z_{3}$, and the constraint (135) now reads

$$
\left|z_{1}\right|^{2}+\left|z_{2}\right|^{2}+\left|z_{3}\right|^{2}-3\left|z_{0}\right|^{2}=t .
$$


The $U(1)$ action on the $z$ s is

$$
\left(z_{0}, z_{1}, z_{2}, z_{3}\right) \rightarrow\left(\mathrm{e}^{-3 \mathrm{i} \alpha} z_{0}, \mathrm{e}^{\mathrm{i} \alpha} z_{1}, \mathrm{e}^{\mathrm{i} \alpha} z_{2}, \mathrm{e}^{\mathrm{i} \alpha} z_{3}\right)
$$

Notice that $z_{1,2,3}$ describe the basis $\mathbb{P}^{2}$, while $z_{0}$ parameterizes the complex direction of the fibre.

Let us now give a description in terms of glued $\mathbf{C}^{3}$ patches. There are three patches $U_{i}$ defined by $z_{i} \neq 0$, for $i=1,2,3$, since at least one of these three co-ordinates must be non-zero in $X$. All of these three patches look like $\mathbf{C}^{3}$. For example, for $z_{3} \neq 0$, we can 'solve' again for $z_{3}$ in terms of the other three unconstrained co-ordinates that then parameterize $\mathbf{C}^{3}: U_{3}=\left(z_{0}, z_{1}, z_{2}\right)$. Similar statements hold for the other two patches. Let us now construct the corresponding degeneration graph. In the $U_{3}=\left(z_{0}, z_{1}, z_{2}\right)$ patch we take as our Hamiltonians

$$
\begin{aligned}
& r_{\alpha}=\left|z_{1}\right|^{2}-\left|z_{0}\right|^{2}, \\
& r_{\beta}=\left|z_{2}\right|^{2}-\left|z_{0}\right|^{2} .
\end{aligned}
$$

The graph of the degenerate fibres in the $r_{\alpha}-r_{\beta}$ plane is the same as in the $\mathbf{C}^{3}$ example, Fig. 12. The third direction in the base, $r_{\gamma}$ is now given by the gauge invariant product $r_{\gamma}=\operatorname{Im}\left(z_{0} z_{1} z_{2} z_{3}\right)$. The same two Hamiltonians $r_{\alpha, \beta}$

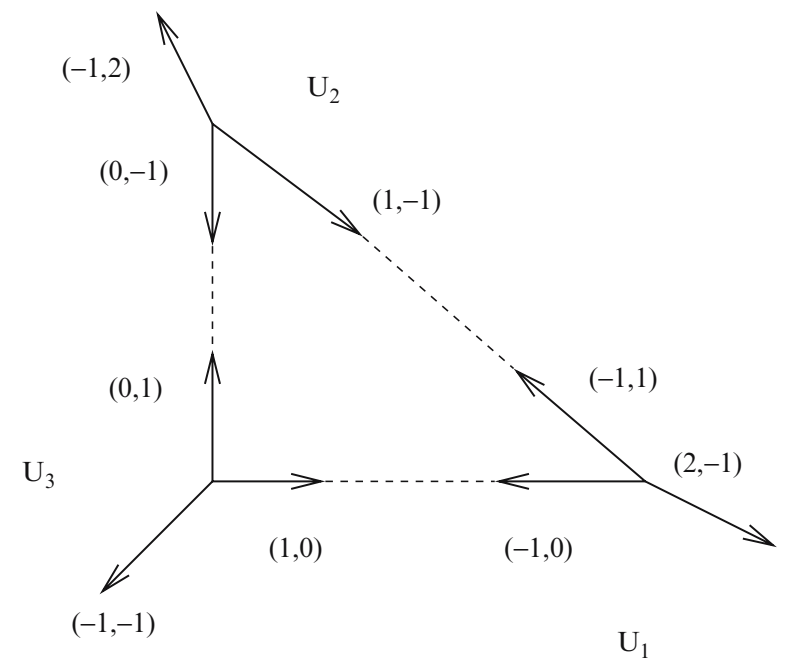

Fig. 14. The graph of $\mathcal{O}(-3) \rightarrow \mathbb{P}^{2}$. This manifold is built out of three $\mathbf{C}^{3}$ patches

generate the action in the $U_{2}=\left(z_{0}, z_{1}, z_{3}\right)$ patch, and we use the constraint (144) to rewrite them as follows: since both $z_{0}$ and $z_{1}$ are co-ordinates of this patch $r_{\alpha}$ does not change. On the other hand, $r_{\beta}$ must be rewritten since $z_{2}$ is not a natural co-ordinate here. We then find: 


$$
\begin{aligned}
& r_{\alpha}=\left|z_{1}\right|^{2}-\left|z_{0}\right|^{2}, \\
& r_{\beta}=t+2\left|z_{0}\right|^{2}-\left|z_{1}\right|^{2}-\left|z_{3}\right|^{2},
\end{aligned}
$$

hence

$$
\mathrm{e}^{\alpha r_{\alpha}+\beta r_{\beta}}:\left(z_{0}, z_{1}, z_{3}\right) \rightarrow\left(\mathrm{e}^{\mathrm{i}(-\alpha+2 \beta)} z_{0}, \mathrm{e}^{\mathrm{i}(\alpha-\beta)} z_{1}, \mathrm{e}^{-\mathrm{i} \beta} z_{3}\right) .
$$

We see from the above that the fibres degenerate over three lines (1) $r_{\alpha}+r_{\beta}=$ $t$, corresponding to $z_{0}=0=z_{3}$, and where a $(-1,1)$ cycle degenerates; $(2)$ there is a line over which a $(-1,2)$ cycle degenerates where $z_{1}=0=z_{3}$, $2 r_{\alpha}+r_{\beta}=t$, and finally, (3) there is a line over which $r_{\alpha}=0$, and a $(0,1)$ cycle degenerates. The $U_{1}$ patch is similar, and we end up with the graph for $\mathcal{O}(-3) \rightarrow \mathbb{P}^{2}$ shown in Fig. 14 .

Example. Lagrangian submanifolds. In order to consider open string amplitudes in the above Calabi-Yau geometries, we have to construct Lagrangian submanifolds providing boundary conditions, as we explained in Sect. 4.4. Let us start by considering the $\mathbf{C}^{3}$ geometry discussed above. In this case, one can easily construct Lagrangian submanifolds following the work of Harvey and Lawson (1982). In terms of the Hamiltonians in (131), we have three types of them:

$$
\begin{array}{ll}
L_{1}: & r_{\alpha}=0, \quad r_{\beta}=r_{1}, \quad r_{\gamma} \geq 0 . \\
L_{2}: & r_{\alpha}=r_{2}, \quad r_{\beta}=0, \quad r_{\gamma} \geq 0 . \\
L_{3}: & r_{\alpha}=r_{\beta}=r_{3}, \quad r_{\gamma} \geq 0
\end{array}
$$

where $r_{i}, i=1,2,3$, are constants. It is not difficult to check that the above submanifolds are indeed Lagrangian (they turn out to be Special Lagrangian as well). In terms of the graph description we developed above, they correspond to points in the edges of the planar graph spanned by $\left(r_{\alpha}, r_{\beta}\right)$, and they project to semi-infinite straight lines on the basis of the fibration, $\mathbf{R}^{3}$, parameterized by $r_{\gamma} \geq 0$. Since they are located at the edges, where one of the circles of the fibration degenerates, they have the topology of $\mathbf{C} \times \mathbf{S}^{1}$.

It is easy to generalize the construction to other toric geometries, like the resolved conifold or local $\mathbb{P}^{2}$ : Lagrangian submanifolds with the topology of $\mathbf{C} \times \mathbf{S}^{1}$ are just given by points on the edges of the planar graphs. Such Lagrangian submanifolds were first considered in the context of open topological string theory by Aganagic and Vafa (2000), and further studied by Aganagic et al. (2002).

\subsection{Examples of Closed String Amplitudes}

Now that we have presented some detailed constructions of Calabi-Yau threefolds, we can come back totopological string amplitudes, or equivalently to Gromov-Witten invariants. The Gromov-Witten invariants of Calabi-Yau threefolds can be computed in a variety of ways. A powerful technique that can be made mathematically rigorous is the localization technique pioneered 
by Kontsevich (1995). For compact Calabi-Yau manifolds, only $N_{g=0, \beta}$ have been computed in detail, but for non-compact, toric Calabi-Yau manifolds localization techniques make it possible to compute $N_{g, \beta}$ for arbitrary genus. We will now present some results for the topological string amplitudes $F_{g}$ of the geometries we described above.

The resolved conifold $\mathcal{O}(-1) \oplus \mathcal{O}(-1) \rightarrow \mathbb{P}^{1}$ has a single Kähler parameter $t$ corresponding to the $\mathbb{P}^{1}$ in the base, and its total free energy is given by

$$
F\left(g_{s}, t\right)=\sum_{d=1}^{\infty} \frac{1}{d\left(2 \sin \frac{d g_{s}}{2}\right)^{2}} Q^{d},
$$

where $Q=\mathrm{e}^{-t}$. We see that the only non-zero Gopakumar-Vafa invariant occurs at degree one and genus zero and is given by $n_{1}^{0}=1$. On the other hand, this model already has an infinite number of non-trivial $N_{g, \beta}$ invariants, which can be obtained by expanding the above expression in powers of $g_{s}$. The above expression was obtained in Gromov-Witten theory by Faber and Pandharipande (2000).

The space $\mathcal{O}(-3) \rightarrow \mathbb{P}^{2}$ also has one single Kähler parameter, corresponding to the hyperplane class of $\mathbb{P}^{2}$. By using the localization techniques of Kontsevich, adapted to the non-compact case, one finds (Chiang et al. 1999; Klemm and Zaslow 2001)

$$
\begin{aligned}
& F_{0}(t)=-\frac{t^{3}}{18}+3 Q-\frac{45 Q^{2}}{8}+\frac{244 Q^{3}}{9}-\frac{12333 Q^{4}}{64} \cdots \\
& F_{1}(t)=-\frac{t}{12}+\frac{Q}{4}-\frac{3 Q^{2}}{8}-\frac{23 Q^{3}}{3}+\frac{3437 Q^{4}}{16} \cdots \\
& F_{2}(t)=\frac{\chi(X)}{5720}+\frac{Q}{80}+\frac{3 Q^{3}}{20}+\frac{514 Q^{4}}{5} \cdots
\end{aligned}
$$

and so on. In (150), $t$ is the Kähler class of the manifold, $Q=\mathrm{e}^{-t}$, and $\chi(X)=2$ is the Euler characteristic of local $\mathbb{P}^{2}$. The first term in $F_{0}$ is proportional to the intersection number $H^{3}$ of the hyperplane class, while the first term in $F_{1}$ is proportional to the intersection number $H \cdot c_{2}(X)$. The first term in $F_{2}$ is the contribution of constant maps.

As we explained above, we can express the closed string amplitudes in terms of Gopakumar-Vafa invariants. Let us introduce a generating functional for integer invariants as follows:

$$
f(z, Q)=\sum_{g, \beta} n_{\beta}^{g} z^{g} Q^{\beta},
$$

where $z$ is a formal parameter. For local $\mathbb{P}^{2}$ one finds

$f(z, Q)=3 Q-6 Q^{2}+(27-10 z) Q^{3}-\left(192-231 z+102 z^{2}-15 z^{3}\right) Q^{4}+\mathcal{O}\left(Q^{5}\right)$. 
It should be mentioned that there is a very powerful method to compute the amplitudes $F_{g}$, namely mirror symmetry. In the mirror symmetric computation, the $F_{g}$ amplitudes are deeply related to the variation of complex structures on the Calabi-Yau manifold (Kodaira-Spencer theory) and can be computed through the holomorphic anomaly equations of Bershadsky et al. (1993, 1994). Gromov-Witten invariants of non-compact, toric Calabi-Yau threefolds have been computed with mirror symmetry by Chiang et al. (1999), Klemm and Zaslow (2001) and Katz et al. (1999).

\section{The Topological Vertex}

\subsection{The Gopakumar-Vafa Duality}

For topological string theory on the resolved conifold, the result in (149) shows that there is only one nontrivial Gopakumar-Vafa invariant. If we now take into account (111), we see that the free energies $F_{g}(t)$ are precisely the resummed functions (33) of Chern-Simons theory, after we identify the string coupling constant $g_{s}$ with the gauge theory coupling constant as in (81), and the Kähler parameter of the resolved conifold is identified with the 't Hooft coupling

$$
t=\mathrm{i} g_{s} N=x N .
$$

Based on this and other evidence, Gopakumar and Vafa (1998b) conjectured that Chern-Simons theory on $\mathbf{S}^{3}$ is equivalent to closed topological string theory on the resolved conifold.

From the point of view of topological string theory, this equivalence only illuminates the resolved conifold geometry, which on the other hand is easy to compute. The fundamental question is: can we use this duality to obtain information about more general Calabi-Yau threefolds? The answer is yes, and the underlying reasoning is heavily based on the idea of geometric transitions, which we won't explain here (see Mariño 2005, for a detailed exposition). This line of reasoning leads directly to the idea of the topological vertex.

\subsection{Framing of Topological Open String Amplitudes}

As we will see, the topological vertex is an open string amplitude, and in order to understand it properly we have to discuss one aspect of open string amplitudes that we have not addressed yet: the framing ambiguity. The framing ambiguity was discovered by Aganagic et al. (2002). They realized that when the boundary conditions are specified by non-compact Lagrangian submanifolds like the ones described in (148), the corresponding topological open string amplitudes are not unambiguously defined: they depend on a choice of an integer (more precisely, one integer for each boundary). For the Lagrangian 
submanifolds considered in Sect. 5, the framing ambiguity can be specified by specifying a vector $f=(p, q)$ attached to the edge where the submanifold is located (see for example Mariño (2005) for a full justification of this). The procedure is illustrated in Fig. 15. It is useful to introduce the symplectic

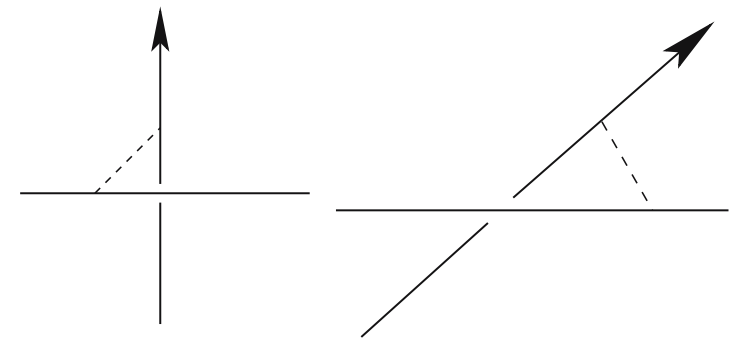

Fig. 15. Specifying a framing

product of two vectors $v=\left(v_{1}, v_{2}\right)$ and $w=\left(w_{1}, w_{2}\right)$ as

$$
v \wedge w=v_{1} w_{2}-v_{2} w_{1}
$$

This product is invariant under $\mathrm{SL}(2, \mathbf{Z})$ transformations. If the original Lagrangian submanifold is located at an edge $v$, the choice of framing has to satisfy

$$
f \wedge v=1 .
$$

Clearly, if $f$ satisfies (155), so does $f-n v$ for any integer $n$. The choice of the integer $n$ is precisely the framing ambiguity found by Aganagic et al. (2002). In the case of the Lagrangian submanifolds of $\mathbf{C}^{3}$ that we constructed in Sect. 5, A particular choice of framing that will be very important in the following is shown in Fig. 16.

What is the effect of a change of framing on open topological string amplitudes? A proposal for this was made by Aganagic et al. (2002) and further studied by Mariño and Vafa (2002), based on the duality with Chern-Simons theory. As pointed out by Ooguri and Vafa (2000), vacuum expectation values of Wilson loops in Chern-Simons theory on $\mathbf{S}^{3}$ compute open string amplitudes. On the other hand, we explained in Sect. 2 that Wilson loop correlation functions depend on a choice of framing. This suggests that the framing ambiguity of Chern-Simons theory corresponds to the ambiguity of topological open string amplitudes that we have just described. This also leads to a very precise prescription to compute the effect of a change of framing for open string amplitudes. Let us consider for simplicity an open string amplitude involving a single Lagrangian submanifold, computed for a framing $f$. If we now consider the framing $f-n v$, the coefficients $Z_{R}$ of the total partition function (120) change as follows 


$$
Z_{R} \rightarrow(-1)^{n \ell(R)} q^{\frac{n \kappa_{R}}{2}} Z_{R},
$$

where $\kappa_{R}$ was defined in (74), and $q=e^{i g_{s}}$. This is essentially the behaviour of Chern-Simons invariants under change of framing spelled out in (75). The extra sign in (156) is crucial to guarantee integrality of the resulting amplitudes, as was verified in Aganagic et al. (2002) and Mariño and Vafa (2002). If the open string amplitudes involves $L$ boundaries, one has to specify $L$ different framings $n_{\alpha}$, and (156) is generalized to

$$
Z_{R_{1} \cdots R_{L}} \rightarrow(-1)^{\sum_{\alpha=1}^{L} n_{\alpha} \ell\left(R_{\alpha}\right)} q^{\sum_{\alpha=1}^{L} n_{\alpha} \kappa_{R_{\alpha}} / 2} Z_{R_{1} \cdots R_{L}} .
$$

\subsection{Definition of the Topological Vertex}

In Sect. 5 we showed that we can construct one Lagrangian submanifold in each of the vertices of the toric diagram of $\mathbf{C}^{3}$. Since each of these submanifolds has the topology of $\mathbf{C} \times \mathbf{S}^{1}$, we can consider the topological open string amplitude associated to this geometry. The total open string partition function will be given by

$$
Z\left(V_{i}\right)=\sum_{R_{1}, R_{2}, R_{3}} C_{R_{1} R_{2} R_{3}} \prod_{i=1}^{3} \operatorname{Tr}_{R_{i}} V_{i}
$$

where $V_{i}$ is a matrix source associated to the $i$-th Lagrangian submanifold. The amplitude $C_{R_{1} R_{2} R_{3}}$ is a function of the string coupling constant $g_{s}$ and, in the genus expansion, it contains information about maps from Riemann surfaces of arbitrary genera into $\mathbf{C}^{3}$ with boundaries on $L_{i}$. This open string amplitude is called the topological vertex, and it is the basic object from which, by gluing, one can obtain closed and open string amplitudes on arbitrary toric geometries. Since the vertex is an open string amplitude, it will depend on a choice of three different framings. As we explained in the previous section, this choice will be given by three different vectors $f_{1}, f_{2}$ and $f_{3}$. Let us see how to introduce this choice.

We saw in Sect. 5 that the $\mathbf{C}^{3}$ geometry can be represented by graphs involving three vectors $v_{i}$. These vectors can be obtained from the set in Fig. 12 by an $\operatorname{SL}(2, \mathbf{Z})$ transformation, and satisfy (134). We will then introduce a topological vertex amplitude $C_{R_{1} R_{2} R_{3}}^{\left(v_{i}, f_{i}\right)}$ that depends on both a choice of three vectors $v_{i}$ for the edges and a choice of three vectors $f_{i}$ for the framings. Due to $(155)$ we require

$$
f_{i} \wedge v_{i}=1
$$

We will orient the edges $v_{i}$ in a clockwise way. Since wedge products are preserved by $\mathrm{SL}(2, \mathbf{Z})$, we also have

$$
v_{2} \wedge v_{1}=v_{3} \wedge v_{2}=v_{1} \wedge v_{3}=1 .
$$


However, not all of these choices give independent amplitudes. First of all, there is an underlying $\mathrm{SL}(2, \mathbf{Z})$ symmetry relating the choices: if $g \in \mathrm{SL}(2, \mathbf{Z})$, then the amplitudes are invariant under

$$
\left(f_{i}, v_{i}\right) \rightarrow\left(g \cdot f_{i}, g \cdot v_{i}\right) .
$$

Moreover, if the topological vertex amplitude $C_{R_{1} R_{2} R_{3}}^{\left(v_{i}, f_{i}\right)}$ is known for a set of framings $f_{i}$, then it can be obtained for any set of the form $f_{i}-n_{i} v_{i}$, and it is given by the general rule (157)

$$
C_{R_{1} R_{2} R_{3}}^{\left(v_{i}, f_{i}-n v_{i}\right)}=(-1)^{\sum_{i} n_{i} \ell\left(R_{i}\right)} q^{\sum_{i} n_{i} \kappa_{R_{i}} / 2} C_{R_{1} R_{2} R_{3}}^{\left(v_{i}, f_{i}\right)},
$$

for all admissible choices of the vectors $v_{i}$. Since any two choices of framing can be related through (160), it is useful to pick a convenient set of $f_{i}$ for any given choice of $v_{i}$, which we will define as the canonical framing of the topological vertex. This canonical framing turns out to be

$$
\left(f_{1}, f_{2}, f_{3}\right)=\left(v_{2}, v_{3}, v_{1}\right) .
$$

Due to the $\operatorname{SL}(2, \mathbf{Z})$ symmetry and the transformation rule (160), any topological vertex amplitude can be obtained from the amplitude computed for a fixed choice of $v_{i}$ in the canonical framing. A useful choice of the $v_{i}$ is $v_{1}=(-1,-1), v_{2}=(0,1), v_{3}=(1,0)$, as in Fig. 12. The vertex amplitude for the canonical choice of $v_{i}$ and in the canonical framing will be simply denoted by $C_{R_{1} R_{2} R_{3}}$. Any other choice of framing will be characterized by framing vectors of the form $f_{i}-n_{i} v_{i}$, and the corresponding vertex amplitude will be denoted by

$$
C_{R_{1} R_{2} R_{3}}^{n_{1}, n_{2}, n_{3}} \text {. }
$$

Notice that $n_{i}=f_{i} \wedge v_{i+1}($ where $i$ runs $\bmod 3)$.

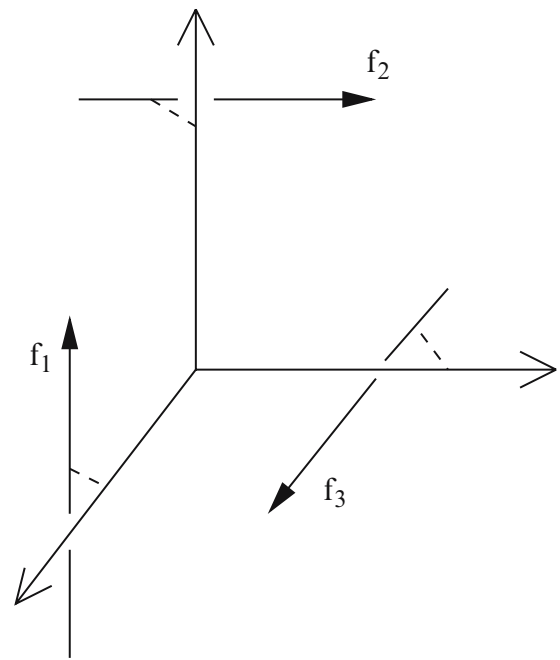

Fig. 16. The canonical choice of framing for the topological vertex 
One of the most important properties of $C_{R_{1} R_{2} R_{3}}$ is its cyclic symmetry. To see this, notice that the $\mathrm{SL}(2, \mathbf{Z})$ transformation $g=T S^{-1}$ takes

$$
\left(v_{i}, f_{i}\right) \rightarrow\left(v_{i+1}, f_{i+1}\right),
$$

where again $i$ runs mod 3 . It then follows that

$$
C_{R_{1} R_{2} R_{3}}=C_{R_{3} R_{1} R_{2}}=C_{R_{2} R_{3} R_{1}} .
$$

Finally, it will sometimes be useful to consider the vertex in the basis of conjugacy classes $C_{\mathbf{k}^{(1)} \mathbf{k}^{(2)} \mathbf{k}^{(3)}}$, which is obtained from $C_{R_{1} R_{2} R_{3}}$ by

$$
C_{\mathbf{k}^{(1)} \mathbf{k}^{(2)} \mathbf{k}^{(3)}}=\sum_{R_{i}} \prod_{i=1}^{3} \chi_{R_{i}}\left(C\left(\mathbf{k}^{(i)}\right)\right) C_{R_{1} R_{2} R_{3}} .
$$

\subsection{Gluing Rules}

We saw in Sect. 5 that any non-compact toric geometry can be encoded in a planar graph that can be obtained by gluing trivalent vertices. It is then natural to expect that the string amplitudes associated to such a diagram can be computed by gluing the open topological string amplitudes associated to the trivalent vertices, in the same way that one computes amplitudes in perturbative quantum field theory by gluing vertices through propagators. This idea was suggested by Aganagic et al. (2004) and Iqbal (2002), and was developed into a complete set of rules by Aganagic et al. (2005). The gluing rules for the topological vertex turn out to be quite simple. Here we will state three rules (for a change of orientation in one edge, for the propagator, and for the matching of framings in the gluing) which make it possible to compute any closed string amplitude on toric, non-compact Calabi-Yau threefolds. They also make it possible to compute open string amplitudes for Lagrangian submanifolds on edges that go to infinity. The case of Lagrangian submanifolds on inner edges is also very easy to analyze, but we refer the reader to the paper by Aganagic, Klemm, Mariño, and Vafa (2005) for the details. A mathematical point of view on the gluing rules can be found in Diaconescu and Florea (2005) and Li et al. (2004):

1) Orientation. Trivalent vertices are glued along their edges, and this corresponds to gluing curves with holes along their boundaries. In order to do that, the boundaries must have opposite orientations. This change of orientation will be represented as an inversion of the edge vector, therefore in gluing the vertices we will have an outgoing edge on one side, say $v_{1}$, and an ingoing 
edge on the other side, $-v_{1}$. It can be shown that the under this operation the topological vertex changes as

$$
C_{R_{1} R_{2} R_{3}} \rightarrow(-1)^{\ell\left(R_{1}\right)} C_{R_{1}^{t} R_{2} R_{3}} .
$$

Of course, a similar equation follows for the other $v_{i}$.

2) Propagator. Since gluing the edges corresponds to gluing curves with holes along their boundaries, we must have matching of the number of holes and winding numbers along the edge. After taking into account the change of orientation discussed above, and after a simple analysis (Aganagic et al. 2005 ), one finds that the propagator for gluing edges with representations $R_{1}$, $R_{2}$ is given by

$$
(-1)^{\ell\left(R_{1}\right)} \mathrm{e}^{-\ell\left(R_{1}\right) t} \delta_{R_{1} R_{2}^{t},}
$$

where $t$ is the Kähler parameter that corresponds to the $\mathbb{P}^{1}$ represented by the gluing edge.

3) Framing. When gluing two vertices, the framings of the two edges involved in the gluing have to match. This means that, in general, we will have to change the framing of one of the vertices. Let us consider the case in which we glue together two vertices with outgoing vectors $\left(v_{i}, v_{j}, v_{k}\right)$ and $\left(v_{i}^{\prime}, v_{j}^{\prime}, v_{k}^{\prime}\right)$, respectively, and let us assume that we glue them through the vectors $v_{i}$, $v_{i}^{\prime}=-v_{i}$. We also assume that both vertices are canonically framed, so that $f_{i}=v_{j}, f_{i}^{\prime}=v_{j}^{\prime}$. In order to match the framings we have to change the framing of, say, $v_{i}^{\prime}$, so that the new framing is $-f_{i}$ (the opposite sign is again due to the change of orientation). There is an integer $n_{i}$ such that $f_{i}^{\prime}-n_{i} v_{i}^{\prime}=-f_{i}$ (since $f_{i} \wedge v_{i}=f_{i}^{\prime} \wedge v_{i}^{\prime}=1, f_{i}+f_{i}^{\prime}$ is parallel to $v_{i}$ ), and it is easy to check that

$$
n_{i}=v_{j}^{\prime} \wedge v_{j} .
$$

The gluing of the two vertex amplitudes is then given by

$$
\sum_{R_{i}} C_{R_{j} R_{k} R_{i}} \mathrm{e}^{-\ell\left(R_{i}\right) t_{i}}(-1)^{\left(n_{i}+1\right) \ell\left(R_{i}\right)} q^{-n_{i} \kappa_{R_{i}} / 2} C_{R_{i}^{t} R_{j}^{\prime} R_{k}^{\prime}},
$$

where we have taken into account the change of orientation in the $\left(v_{i}^{\prime}, v_{j}^{\prime}, v_{k}^{\prime}\right)$ to perform the gluing, and $t_{i}$ is the Kähler parameter associated to the edge.

Given then a planar trivalent graph representing a non-compact CalabiYau manifold, we can compute the closed string amplitude as follows: we give a presentation of the graph in terms of vertices glued together, as we did in Sect.5. We associate the appropriate amplitude to each trivalent vertex (labelled by representations), and we use the above gluing rules. The edges that go to infinity carry the trivial representation, and we finally sum over all possible representations along the inner edges. The resulting quantity is the total partition function $Z_{\text {closed }}=e^{F}$ for closed string amplitudes. 


\subsection{Explicit Expression for the Topological Vertex}

Once we have defined the topological vertex, we need an explicit expression for it. This turns out to be a difficult problem which can however be explicitly solved. The basic idea is to use an extension of the Gopakumar-Vafa duality to open string amplitudes. As shown by Ooguri and Vafa (2000), the duality between Chern-Simons theory and the resolved conifold leads to a correspondence between Chern-Simons invariants for knots in $\mathbf{S}^{3}$ and open topological string amplitudes with Lagrangian boundary conditions in the resolved conifold. This idea applies in principle to Lagrangian submanifolds in the resolved conifold, but one can extend it to other contexts, and in particular to the configuration considered above involving three Lagrangian submanifolds in $\mathbf{C}^{3}$.

It turns out that the open topological string amplitude for the three Lagrangian submanifolds in $\mathbf{C}^{3}$ can be written by using only the ChernSimons invariant of the Hopf link that we studied in Sect. 2. Let $\mathcal{W}_{R_{1} R_{2}}$ is the Hopf link invariant defined in (54) and evaluated in (62). We now consider the limit

$$
W_{R_{1} R_{2}}=\lim _{t \rightarrow \infty} \mathrm{e}^{-\frac{\ell\left(R_{1}\right)+\ell\left(R_{2}\right)}{2} t} \mathcal{W}_{R_{1} R_{2}}
$$

This limit exists, since $\mathcal{W}_{R_{1} R_{2}}$ is of the form $\lambda^{\frac{\ell\left(R_{1}\right)+\ell\left(R_{2}\right)}{2}} W_{R_{1} R_{2}}+\mathcal{O}\left(\mathrm{e}^{-t}\right)$ (recall that $\left.\lambda=\mathrm{e}^{t}\right)$. The quantity $W_{R_{1} R_{2}}$, which is the 'leading' coefficient of the Hopf link invariant (54), is the building block of the topological vertex amplitude. It is a rational function of $q^{ \pm \frac{1}{2}}$, therefore it only depends on the string coupling constant. We will also denote $W_{R}=W_{R 0}$. The limit (165) was first considered by Aganagic et al. (2004). The final expression for the vertex, in the canonical framing defined above, is

$$
C_{R_{1} R_{2} R_{3}}=q^{\frac{\kappa_{R_{2}}+\kappa_{R_{3}}}{2}} \sum_{Q_{1}, Q_{3}, Q} N_{Q Q_{1}}^{R_{1}} N_{Q Q_{3}}^{R_{3}^{t}} \frac{W_{R_{2}^{t} Q_{1}} W_{R_{2} Q_{3}}}{W_{R_{2}}},
$$

where $N_{R_{1} R_{2}}^{R}$ is the Littlewood-Richardson coefficient which gives the multiplicity of $R$ in the tensor product $R_{1} \otimes R_{2}$.

Let us now give some more explicit formulae for the vertex. The basic ingredient in (166) is the quantity $W_{R_{1} R_{2}}$ defined in (165). Using (62) it is possible to give an explicit expression for $W_{R_{1} R_{2}}$ that is useful in computations. It is easy to see that the leading coefficient of $\lambda$ in (62) is obtained by taking the leading coefficient of $\lambda$ in $\operatorname{dim}_{q} R_{2}$ and the $\lambda$-independent piece in (65). The generating function of elementary symmetric polynomials (63) then becomes

$$
S(t) \prod_{j=1}^{c_{R}} \frac{1+q^{l_{j}^{R}-j} t}{1+q^{-j} t},
$$


where

$$
S(t)=\prod_{j=1}^{\infty}\left(1+q^{-j} t\right)=1+\sum_{r=1}^{\infty} \frac{q^{-\frac{r(r+1)}{2}} t^{r}}{\prod_{m=1}^{r}[m]}
$$

Notice that (167) is the generating function of elementary symmetric polynomials for an infinite number of variables given by $x_{j}=q^{l_{j}^{R_{1}}-j}, j=1,2, \cdots$. One then deduces that the $\lambda \rightarrow \infty$ limit of $q^{\ell\left(R_{1}\right) / 2} s_{R_{1}}\left(x_{i}=q^{l_{i}^{R_{2}}-i}\right)$ is given by the Schur polynomial

$$
s_{R_{1}}\left(x_{i}=q^{l_{i}^{R_{2}}-i+\frac{1}{2}}\right),
$$

which now involves an infinite number of variables $x_{i}$. This finally leads to the following expression for $W_{R_{1} R_{2}}$ :

$$
W_{R_{1} R_{2}}(q)=s_{R_{1}}\left(x_{i}=q^{l_{i}^{R_{2}}-i+\frac{1}{2}}\right) s_{R_{2}}\left(x_{i}=q^{-i+\frac{1}{2}}\right) .
$$

We will also write this as

$$
W_{R_{1} R_{2}}(q)=s_{R_{1}}\left(q^{\rho+l^{R_{2}}}\right) s_{R_{2}}\left(q^{\rho}\right),
$$

where the arguments of the Schur functions indicate the above values for the polynomial variables $x_{i}$. Using (170) one can write (166) in terms of skew Schur polynomials (Okounkov et al. 2003):

$$
C_{R_{1} R_{2} R_{3}}=q^{\frac{1}{2}\left(\kappa_{R_{2}}+\kappa_{R_{3}}\right)} s_{R_{2}^{t}}\left(q^{\rho}\right) \sum_{Q} s_{R_{1} / Q}\left(q^{\ell\left(R_{2}^{t}\right)+\rho}\right) s_{R_{3}^{t} / Q}\left(q^{\ell\left(R_{2}\right)+\rho}\right) .
$$

\subsection{Applications}

We will now present some examples of computation of topological string amplitudes by using the topological vertex.

Example. Resolved conifold. The toric diagram for the resolved conifold geometry is depicted in Fig. 13. Our rules give immediately:

$$
Z_{\mathbb{P}^{1}}=\sum_{R} C_{00 R^{t}}(-1)^{\ell(R)} \mathrm{e}^{-\ell(R) t} C_{R 00}
$$

Since $C_{R 00}=W_{R}=s_{R}\left(x_{i}=q^{-i+\frac{1}{2}}\right)$, we can use well-known formulae for Schur polynomials to obtain

$$
Z_{\mathbb{P}^{1}}=\exp \left\{-\sum_{d=1}^{\infty} \frac{\mathrm{e}^{-d t}}{d\left(q^{\frac{d}{2}}-q^{-\frac{d}{2}}\right)^{2}}\right\}
$$

in agreement with the known result (149). 
Example. Local $\mathbb{P}^{2}$. The toric diagram is depicted in Fig. 14. Using again the rules explained above, we find the total partition function

$$
Z_{\mathbb{P}^{2}}=\sum_{R_{1}, R_{2}, R_{3}}(-1)^{\sum_{i} \ell\left(R_{i}\right)} \mathrm{e}^{-\sum_{i} \ell\left(R_{i}\right) t} q^{-\sum_{i} \kappa_{R_{i}}} C_{0 R_{2}^{t} R_{3}} C_{0 R_{1}^{t} R_{2}} C_{0 R_{3}^{t} R_{1}}
$$

where $t$ is the Kähler parameter corresponding to the hyperplane class in $\mathbb{P}^{2}$. Using that $C_{0 R_{2} R_{3}^{t}}=W_{R_{2} R_{3}} q^{-\kappa_{R_{3}} / 2}$ one recovers the expression for $Z_{\mathbb{P}^{2}}$ first obtained by Aganagic et al. (2004). Notice that the free energy has the structure

$$
F_{\mathbb{P}^{2}}=\log \left\{1+\sum_{\ell=1}^{\infty} a_{\ell}(q) \mathrm{e}^{-\ell t}\right\}=\sum_{\ell=1}^{\infty} a_{\ell}^{(c)}(q) e^{-\ell t}
$$

The coefficients $a_{\ell}(q), a_{\ell}^{(c)}(q)$ can be easily obtained in terms of $W_{R_{1} R_{2}}$. One finds, for example,

$$
\begin{aligned}
& a_{1}^{(c)}(q)=a_{1}(q)=-\frac{3}{\left(q^{\frac{1}{2}}-q^{-\frac{1}{2}}\right)^{2}}, \\
& a_{2}^{(c)}(q)=\frac{6}{\left(q^{\frac{1}{2}}-q^{-\frac{1}{2}}\right)^{2}}+\frac{1}{2} a_{1}\left(q^{2}\right) .
\end{aligned}
$$

If we compare to (110) and take into account the effects of multicovering, we find the following values for the Gopakumar-Vafa invariants of $\mathcal{O}(-3) \rightarrow \mathbb{P}^{2}$ :

$$
\begin{array}{cc}
n_{1}^{0}=3, & n_{1}^{g}=0 \text { for } g>0, \\
n_{2}^{0}=-6, & n_{2}^{g}=0 \text { for } g>0,
\end{array}
$$

in agreement with the results listed in (152). In fact, one can go much further with this method and compute the Gopakumar-Vafa invariants to high degree. We again see that the use of exact results in Chern-Simons theory leads to the topological string amplitudes to all genera. A complete listing of the Gopakumar-Vafa invariants up to degree 12 can be found in Aganagic et al. (2004). The partition function (174) can also be computed in Gromov-Witten theory by using localization techniques, and one finds indeed the same result (Zhou 2003).

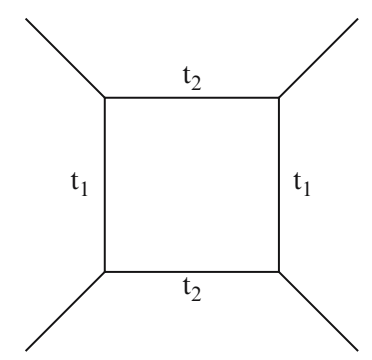

Fig. 17. The toric diagram of local $\mathbb{P}^{1} \times \mathbb{P}^{1}$ 
Example. Local $\mathbb{P}^{1} \times \mathbb{P}^{1}$. The local $\mathbb{P}^{1} \times \mathbb{P}^{1}$ geometry is the non-compact Calabi-Yau manifold given by the four-manifold $\mathbb{P}^{1} \times \mathbb{P}^{1}$ together with its anti-canonical bundle. It also admits a symplectic quotient description of the form (137), this time with $N=2$ and two Kähler parameters $t_{1}, t_{2}$. The charges $Q_{1,2}^{j}, j=1, \cdots, 5$ can be grouped into two vectors

$$
\begin{aligned}
& Q_{1}=(-2,1,1,0,0), \\
& Q_{2}=(-2,0,0,1,1) .
\end{aligned}
$$

The toric diagram for this geometry can be easily worked out from this description, and it is represented in Fig. 17. Using the gluing rules we find the closed string partition function

$$
\begin{aligned}
Z_{\mathbb{P}^{1} \times \mathbb{P}^{1}}= & \sum_{R_{i}} \mathrm{e}^{-\left(\ell\left(R_{1}\right)+\ell\left(R_{3}\right)\right) t_{1}-\left(\ell\left(R_{2}\right)+\ell\left(R_{4}\right)\right) t_{2}} q^{\sum_{i} \kappa_{R_{i}} / 2} \\
& \times C_{0 R_{4} R_{1}^{t}} C_{0 R_{1} R_{2}^{t}} C_{0 R_{2} R_{3}^{t}} C_{0 R_{3} R_{4}^{t}} .
\end{aligned}
$$

This amplitude can be written as

$$
\begin{aligned}
Z_{\mathbb{P}^{1} \times \mathbb{P}^{1}}= & \sum_{R_{i}} \mathrm{e}^{-\left(\ell\left(R_{1}\right)+\ell\left(R_{3}\right)\right) t_{1}-\left(\ell\left(R_{2}\right)+\ell\left(R_{4}\right)\right) t_{2}} \\
& \times W_{R_{4} R_{1}} W_{R_{1} R_{2}} W_{R_{2} R_{3}} W_{R_{3} R_{4}} .
\end{aligned}
$$

This is the expression first obtained by Aganagic et al. (2004). It has been shown to agree with Gromov-Witten theory by Zhou (2003).

Example. The closed topological vertex. Consider the Calabi-Yau geometry whose toric diagram is depicted in Fig. 18. It contains three $\mathbb{P}^{1}$ touching at a single point. The local Gromov-Witten theory of this geometry was studied by Bryan and Karp (2005), who called it the closed topological vertex, and also by Karp et al. (2005). The vertex rules give the following expression for the total partition function:

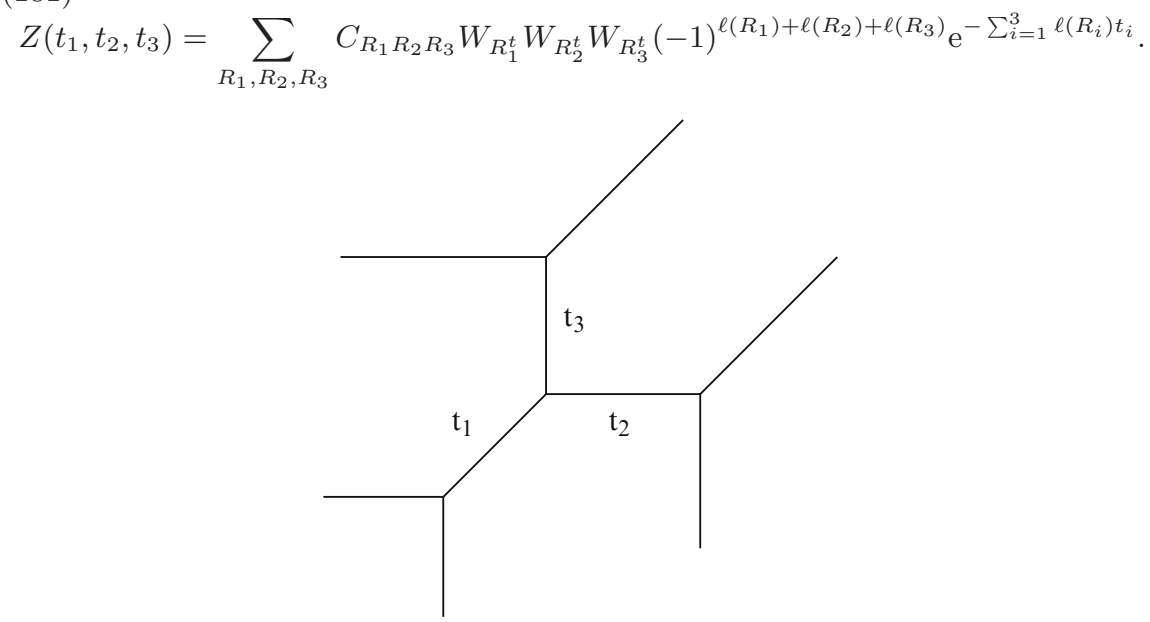

Fig. 18. The toric diagram of the closed topological vertex 
It turns out that this can be evaluated in closed form (Karp et al. 2005)

$$
\begin{aligned}
& Z\left(t_{1}, t_{2}, t_{3}\right)=\exp \left(-\sum_{d=1}^{\infty} \frac{1}{d\left(q^{\frac{d}{2}}-q^{-\frac{d}{2}}\right)}\left(\mathrm{e}^{-d t_{1}}+\mathrm{e}^{-d t_{2}}+\mathrm{e}^{-d t_{3}}\right.\right. \\
& \left.\left.-\mathrm{e}^{-d\left(t_{1}+t_{2}\right)}-\mathrm{e}^{-d\left(t_{1}+t_{3}\right)}-\mathrm{e}^{-d\left(t_{2}+t_{3}\right)}+\mathrm{e}^{-d\left(t_{1}+t_{2}+t_{3}\right)}\right)\right),
\end{aligned}
$$

in agreement with the algebro-geometric results of Bryan and Karp (2005). Notice from the above expression that there is only a finite number of nonvanishing Gopakumar-Vafa invariants for the above geometry.

\section{A Symmetric Polynomials}

In this brief Appendix we summarize some useful ingredients of the elementary theory of symmetric functions. A standard reference is Macdonald (1995).

Let $x_{1}, \cdots, x_{N}$ denote a set of $N$ variables. The elementary symmetric polynomials in these variables, $e_{m}(x)$, are defined as:

$$
e_{m}(x)=\sum_{i_{1}<\cdots<i_{m}} x_{i_{1}} \cdots x_{i_{m}} .
$$

The generating function of these polynomials is given by

$$
E(t)=\sum_{m \geq 0} e_{m}(x) t^{m}=\prod_{i=1}^{N}\left(1+x_{i} t\right) .
$$

The complete symmetric function $h_{m}$ can be defined in terms of its generating function

$$
H(t)=\sum_{m \geq 0} h_{m} t^{m}=\prod_{i=1}^{N}\left(1-x_{i} t\right)^{-1},
$$

and one has

$$
E(t) H(-t)=1
$$

The products of elementary symmetric polynomials and of complete symmetric functions provide two different basis for the symmetric functions of $N$ variables.

Another basis is given by the Schur polynomials, $s_{R}(x)$, which are labelled by representations $R$. We will always express these representations in terms of Young tableaux, so $R$ is given by a partition $\left(l_{1}, l_{2}, \cdots, l_{c_{R}}\right)$, where $l_{i}$ is the number of boxes of the $i$-th row of the tableau, and we have $l_{1} \geq l_{2} \geq \cdots \geq l_{c_{R}}$. 
The total number of boxes of a tableau will be denoted by $\ell(R)=\sum_{i} l_{i}$. The Schur polynomials are defined as quotients of determinants,

$$
s_{R}(x)=\frac{\operatorname{det} x_{j}^{l_{i}+N-i}}{\operatorname{det} x_{j}^{N-i}} .
$$

They can be written in terms of the symmetric polynomials $e_{i}\left(x_{1}, \cdots, x_{N}\right)$, $i \geq 1$, as follows:

$$
s_{R}=\operatorname{det} M_{R},
$$

where

$$
M_{R}^{i j}=\left(e_{l_{i}^{t}+j-i}\right) .
$$

$M_{R}$ is an $r \times r$ matrix, with $r=c_{R^{t}}$, and $R^{t}$ denotes the transposed Young tableau with row lengths $l_{i}^{t}$. To evaluate $s_{R}$ we put $e_{0}=1, e_{k}=0$ for $k<0$. The expression (188) is known as the Jacobi-Trudi identity.

A third set of symmetric functions is given by the Newton polynomials $P_{\mathbf{k}}(x)$. These are labelled by vectors $\mathbf{k}=\left(k_{1}, k_{2}, \cdots\right)$, where the $k_{j}$ are nonnegative integers, and are defined as

$$
P_{\mathbf{k}}(x)=\prod_{j}^{p} P_{j}^{k_{j}}(x),
$$

where

$$
P_{j}(x)=\sum_{i=1}^{N} x_{i}^{j}
$$

are power sums. The Newton polynomials are homogeneous of degree $\ell=$ $\sum_{j} j k_{j}$ and give a basis for the symmetric functions in $x_{1}, \cdots, x_{N}$ with rational coefficients. They are related to the Schur polynomials through the Frobenius formula

$$
P_{\mathbf{k}}(x)=\sum_{R} \chi_{R}(C(\mathbf{k})) s_{R}(x),
$$

where the sum is over all tableaux such that $\ell(R)=\ell$.

\section{References}

1. Aganagic, M., Klemm, A., Mariño, M., and Vafa, C. (2005). The topological vertex. Commun. Math. Phys. 254, 425-478; eprint hep-th/0305132.

2. Aganagic, M., Klemm, A., and Vafa, C. (2002). Disk instantons, mirror symmetry and the duality web. Z. Naturforsch. A 57, 1-28; eprint hep-th/0105045. 
3. Aganagic, M., Mariño, M., and Vafa, C. (2004). All loop topological string amplitudes from Chern-Simons theory. Commun. Math. Phys. 247, 467-512; eprint hep-th/0206164.

4. Aganagic, M. and Vafa, C. (2000). Mirror symmetry, D-branes and counting holomorphic discs. Eprint hep-th/0012041.

5. Aspinwall, P. S., and Morrison, D. R. (1993). Topological field theory and rational curves. Commun. Math. Phys. 151, 245-62; eprint hep-th/9110048.

6. Atiyah, M. (1990). On framings of three-manifolds. Topology 29, 1-7.

7. Axelrod, S. and Singer, I. (1992). Chern-Simons perturbation theory. In Catto, Sultan (ed.) et al., Differential geometric methods in theoretical physics, World Scientific, Singapore, pp. 3-45; eprint hep-th/9110056.

8. Bar-Natan, D. (1995). On the Vassiliev knot invariants. Topology 34, 423-72.

9. Bershadsky, M., Cecotti, S., Ooguri, H., and Vafa, C. (1993). Holomorphic anomalies in topological field theories. Nucl. Phys. B 405, 279-304; eprint hepth/9302103.

10. Bershadsky, M., Cecotti, S., Ooguri, H., and Vafa, C. (1994). Kodaira-Spencer theory of gravity and exact results for quantum string amplitudes. Commun. Math. Phys. 165, 311-428; eprint hep-th/9309140.

11. Bryan, J. and Karp, D. (2005). The closed topological vertex via the Cremona transform. J. Algebraic Geom. 14, 529-42; eprint math.AG/0311208.

12. Bryan, J. and Pandharipande, R. (2001). BPS states of curves in Calabi-Yau 3-folds. Geom. Topol. 5, 287-318; eprint math.AG/0009025.

13. Camperi, M., Levstein, F., and Zemba, G. (1990). The large $N$ limit of ChernSimons theory. Phys. Lett. B 247, 549-54.

14. Candelas, P., De La Ossa, X. C., Green, P., and Parkes, L. (1991). A pair of Calabi-Yau manifolds as an exactly soluble superconformal theory. Nucl. Phys. B 359, 21-74.

15. Chiang, T. M., Klemm, A., Yau, S. T., and Zaslow, E. (1999). Local mirror symmetry: Calculations and interpretations. Adv. Theor. Math. Phys. 3, 495565; eprint hep-th/9903053.

16. Correale, R. and Guadagnini, E. (1994). Large N Chern-Simons field theory. Phys. Lett. B 337, 80-5.

17. Cox, D. and Katz, S. 1999. Mirror symmetry and algebraic geometry, American Mathematical Society, Providence.

18. Cvitanovic, P. (1976). Group theory for Feynman diagrams in nonabelian gauge theories. Phys. Rev. D 14, 1536-53.

19. Cvitanovic, P. (2004). Group theory. Birdtracks, Lie's, and exceptional groups. In http://www.cns.gatech.edu/GroupTheory.

20. Diaconescu, D. E. and Florea, B. (2005). Localization and gluing of topological amplitudes. Commun. Math. Phys. 257, 119-49; eprint hep-th/0309143.

21. Di Francesco, P., Mathieu, P., and Sénéchal, D. (1997). Conformal field theory, Springer-Verlag, New York.

22. Dijkgraaf, R. (1995). Perturbative topological field theory. In String theory, gauge theory and quantum gravity '93, 189-227, World Scientific.

23. Elitzur, S., Moore, G., Schwimmer, A., and Seiberg, N. (1989). Remarks on the canonical quantization of the Chern-Simons-Witten theory. Nucl. Phys. B 326, 108-34.

24. Faber, C. and Pandharipande, R. (2000). Hodge integrals and Gromov-Witten theory. Invent. Math. 139, 173-99; eprint math.AG/9810173. 
25. Freed, D. S. and Gompf, R. E. (1991). Computer calculation of Witten's threemanifold invariant. Commun. Math. Phys. 141, 79-117.

26. Freyd, P., Yetter, D., Hoste, J., Lickorish, W.B.R., Millett, K., and Ocneanu, A.A. (1985). A new polynomial invariant of knots and links. Bull. Am. Math. Soc. (N.S.) 12, 239-46.

27. Fulton, W. and Harris, J. (1991). Representation theory. A first course. SpringerVerlag, New York.

28. Gopakumar, R., and Vafa, C. (1998a). M-theory and topological strings, I. Eprint hep-th/9809187.

29. Gopakumar, R. and Vafa, C. (1998b). M-theory and topological strings, II. Eprint hep-th/9812127.

30. Gopakumar, R. and Vafa, C. (1999). On the gauge theory/geometry correspondence. Adv. Theor. Math. Phys. 3, 1415-43; eprint hep-th/9811131.

31. Graber, T. and Zaslow, E. (2002). Open-string Gromov-Witten invariants: Calculations and a mirror "theorem". Contemp. Math. 310, 107-121; eprint hepth/0109075.

32. Griffiths, P. and Harris, J. (1977). Principles of algebraic geometry, John Wiley and Sons, New York.

33. Guadagnini, E., Martellini, M., and Mintchev, M. (1990). Wilson lines in ChernSimons theory and link invariants. Nucl. Phys. B 330, 575-607.

34. Harris, J. and Morrison, I. (1998). Moduli of curves. Springer-Verlag, New York.

35. Harvey, R. and Lawson, H.B. (1982). Calibrated geometries. Acta Math. 148, $47-157$.

36. Hori, K., Katz, S., Klemm, A., Pandharipande, R., Thomas, R., Vafa, C., Vakil, R., and Zaslow, E. (2003). Mirror symmetry. American Mathematical Society, Providence.

37. Iqbal, A. (2002). All genus topological string amplitudes and 5-brane webs as Feynman diagrams. Eprint hep-th/0207114.

38. Jeffrey, L. C. (1992). Chern-Simons-Witten invariants of lens spaces and torus bundles, and the semi-classical approximation. Commun. Math. Phys. 147, 563604.

39. Karp, D., Liu, C.-C. M., and Mariño, M. (2005). The local Gromov-Witten invariants of configurations of rational curves. Eprint math.AG/0506488.

40. Katz, S., Klemm, A., and Vafa, C. (1999). M-theory, topological strings and spinning black holes. Adv. Theor. Math. Phys. 3, 1445-537; eprint hep-th/9910181.

41. Katz, S., and Liu, C.-C. (2002). Enumerative geometry of stable maps with Lagrangian boundary conditions and multiple covers of the disc. Adv. Theor. Math. Phys. 5, 1-49; eprint math.ag/0103074.

42. Klemm, A. and Zaslow, E. (2001). Local mirror symmetry at higher genus. In Winter School on Mirror Symmetry, Vector bundles and Lagrangian Submanifolds, American Mathematical Society, Providence, p. 183-207; eprint hepth/9906046.

43. Kontsevich, M. (1995). Enumeration of rational curves via torus actions. Prog. Math. 129, 335-68; eprint hep-th/9405035.

44. Labastida, J.M.F. (1999). Chern-Simons theory: ten years after. In Trends in Theoretical Physics II, H. Falomir, R. E. Gamboa and F. A. Schaposnik, eds., AIP Conference Proceedings, Vol. 484, p. 1-40; eprint hep-th/9905057.

45. Labastida, J. M. F. and Ramallo, A. V. (1989). Operator formalism for ChernSimons theories. Phys. Lett. B 227, 92-102. 
46. Le, T. T. Q., Murakami, J., and Ohtsuki, T. (1998). On a universal perturbative invariant of 3-manifolds. Topology 37, 539-74.

47. Li, J., Liu, C.-C. M., Liu, K., and Zhou, J. (2004). A mathematical theory of the topological vertex. Eprint math.AG/0408426.

48. Li, J. and Song, Y. S. (2002). Open string instantons and relative stable morphisms. Adv. Theor. Math. Phys. 5, 67-91; eprint hep-th/0103100.

49. Lickorish, W. B. R. (1998). An introduction to knot theory, Springer-Verlag, New York.

50. Macdonald, I.G. (1995). Symmetric functions and Hall polynomials, 2nd edition, Oxford University Press, Oxford.

51. Mariño, M. (2005). Chern-Simons theory, matrix models, and topological strings. Oxford University Press.

52. Mariño, M. and Vafa, C. (2002). Framed knots at large N. Contemp. Math. 310, 185-204; eprint hep-th/0108064.

53. Maulik, D., Nekrasov, N., Okounkov, A., and Pandharipande, R. (2003). Gromov-Witten theory and Donaldson-Thomas theory. Eprint math.AG/ 0312059 .

54. Mayr, P. (2002). Summing up open string instantons and $\mathcal{N}=1$ string amplitudes. Eprint hep-th/0203237.

55. Morton, H.R. and Lukac, S. G. (2003). The HOMFLY polynomial of the decorated Hopf link. J. Knot Theory Ramifications 12, 395-416; eprint math.GT/0108011.

56. Ohtsuki, T. (2002). Quantum invariants. World Scientific, Singapore.

57. Okounkov, A., Reshetikhin, N., and Vafa, C. (2003). Quantum Calabi-Yau and classical crystals. Eprint hep-th/0309208.

58. Ooguri, H. and Vafa, C. (2000). Knot invariants and topological strings. Nucl. Phys. B 577, 419-38; eprint hep-th/9912123.

59. Ooguri, H. and Vafa, C. (2002). Worldsheet derivation of a large $N$ duality. Nucl. Phys. B 641, 3-34; eprint hep-th/0205297.

60. Periwal, V. (1993). Topological closed string interpretation of Chern-Simons theory. Phys. Rev. Lett. 71, 1295-8; eprint hep-th/9305115.

61. Polyakov, A. M. (1988). Fermi-Bose transmutations induced by gauge fields. Mod. Phys. Lett. A 3, 325-8.

62. Prasolov, V. V. and Sossinsky, A. B. (1997). Knots, links, braids and 3manifolds, American Mathematical Society, Providence.

63. Rozansky, L. (1995). A large $k$ asymptotics of Witten's invariant of Seifert manifolds. Commun. Math. Phys. 171, 279-322; eprint hep-th/9303099.

64. Rozansky, L. and Witten, E. (1997). Hyper-Kähler geometry and invariants of three-manifolds. Selecta Math. 3, 401-58; eprint hep-th/9612216.

65. Sawon, J. (2004). Rozansky-Witten invariants of hyperKähler manifolds. Eprint math.DG/0404360.

66. Schwarz, A. (1987). New topological invariants arising in the theory of quantized fields. Baku International Topological Conference, Abstracts (Part 2) Baku.

67. 't Hooft, G. (1974). A planar diagram theory for strong interactions. Nucl. Phys. B 72 461-73.

68. Witten, E. (1989). Quantum field theory and the Jones polynomial. Commun. Math. Phys. 121, 351-99.

69. Witten, E. (1993). Phases of $\mathcal{N}=2$ theories in two dimensions. Nucl. Phys. $B$ 403, 159-222; eprint hep-th/9301042. 
70. Witten, E. (1995). Chern-Simons gauge theory as a string theory. Prog. Math. 133, 637-78; eprint hep-th/9207094.

71. Zhou, J. (2003). Localizations on moduli spaces and free field realizations of Feynman rules. Eprint math.AG/0310283. 